\title{
Vehicle Stability Control Strategy Based on Recognition of Driver Turning Intention for Dual-Motor Drive Electric Vehicle
}

\author{
Shu Wang $\mathbb{D}^{\mathrm{D}}$, Xuan Zhao ${ }^{\mathrm{D}}$, and Qiang Yu $\mathbb{D}$ \\ School of Automobile, Chang'an University, Xi'an 710064, China \\ Correspondence should be addressed to Shu Wang; shuwang@chd.edu.cn and Xuan Zhao; zhaoxuan@chd.edu.cn
}

Received 9 July 2019; Revised 24 November 2019; Accepted 17 December 2019; Published 13 January 2020

Academic Editor: Luis J. Yebra

Copyright (c) 2020 Shu Wang et al. This is an open access article distributed under the Creative Commons Attribution License, which permits unrestricted use, distribution, and reproduction in any medium, provided the original work is properly cited.

\begin{abstract}
Vehicle stability control should accurately interpret the driving intention and ensure that the actual state of the vehicle is as consistent as possible with the desired state. This paper proposes a vehicle stability control strategy, which is based on recognition of the driver's turning intention, for a dual-motor drive electric vehicle. A hybrid model consisting of Gaussian mixture hidden Markov (GHMM) and Generalized Growing and Pruning RBF (GGAP-RBF) neural network is constructed to recognize the driver turning intention in real time. The turning urgency coefficient, which is computed on the basis of the recognition results, is used to establish a modified reference model for vehicle stability control. Then, the upper controller of the vehicle stability control system is constructed using the linear model predictive control theory. The minimum of the quadratic sum of the working load rate of the vehicle tire is taken as the optimization objective. The tire-road adhesion condition, performance of the motor and braking system, and state of the motor are taken as constraints. In addition, a lower controller is established for the vehicle stability control system, with the task of optimizing the allocation of additional yaw moment. Finally, vehicle tests were carried out by conducting double-lane change and single-lane change experiments on a platform for dual-motor drive electric vehicles by using the virtual controller of the A\&D5435 hardware. The results show that the stability control system functions appropriately using this control strategy and effectively improves the stability of the vehicle.
\end{abstract}

\section{Introduction}

Vehicle stability control, which relies on the antilock braking system (ABS) and traction control system (TCS) of a vehicle, plays a significant role in preventing single-vehicle accidents caused by vehicle instability [1]. The mechanical structure, dynamic characteristics, response characteristics, and actuator complexity of a dual-motor drive electric vehicle differ significantly from those of a single-drive vehicle. Therefore, dual-motor drive electric vehicles also require an efficient and stable stability control system.

Depending on the system structure, two types of vehicle stability system controllers are used: centralized and hierarchical. Compared with the centralized controller, the hierarchical controller system has superior extendibility and fault tolerance and allows more convenient system maintenance and debugging $[2,3]$. Furthermore, the much higher level of integration of the vehicle electrification chassis makes it possible to realize coordination control with other systems such as the $X$-wire control system and active safety systems, e.g., electric control braking (ECB), electric brake force distribution (EBD), and active front steering (AFS).

Based on the current state of the vehicle and the driver's intention, the upper layer of the hierarchical vehicle stability controller makes decision regarding the additional yaw moment to restore the stable state of the vehicle. Zhang and Wang proposed a vehicle stability control system using generalized proportion integration (PI) control [4]. Wang et al. developed a stability control strategy based on an integral separation PID controller to eliminate integral accumulation of the control system [5]. Zhao et al. designed a vehicle stability control system based on a T-S fuzzy model $[6,7]$. Some scholars also adopted robust control to improve the influence of uncertain factors such as system loss, tire 
load fluctuation, and side wind [8-12]. However, the PID algorithm guarantees neither optimal control of the system nor its control stability. The rules of fuzzy control are established on the basis of a large number of experiments and expert experience and need to be adjusted according to the driving environment, which is both high time and economic consuming. The most notable disadvantage of slide mode control is chattering, which occurs when the system approaches the slide mode surface and which can only be reduced but not eliminated. Model predictive control (MPC) can directly consider actuator constraints and system state constraints during controller design. In addition, MPC can effectively control a multiobjective constrained system in complex engineering systems and has strong portability [13]. Jalali et al. proposed a vehicle stability controller using MPC [14-17]. And, the desired yaw rate and sideslip angle were obtained through the driver steering wheel angle.

The lower layer of a vehicle stability controller selects the appropriate actuator and reallocates the additional yaw moment based on certain rules. At present, the most commonly used method relies on two allocation strategies: rule-based and optimization theory-based [18]. The increasing actuators and growing complexity of the electronic control systems of vehicles have resulted in the allocation rules becoming more complex; hence, the allocation accuracy cannot be guaranteed to the same extent as before. Therefore, the optimization theory-based allocation strategy is more suitable.

The allocation strategy based on optimization theory usually takes the slip rate of the tire, the minimum of the sum of the working load of the tire, and the minimum of the sum of the longitudinal force of the tire as the optimization objectives. At the same time, the optimization strategy considering the actuator failure and energy economy are gradually put forward. Yin proposed the additional yaw moment and traction force distribution strategy for the purpose of minimizing the tire load [19]. Zhai et al. proposed an average torque distribution strategy, tire-dynamic-load-based torque distribution strategy, and minimum-objective-function-based optimal torque distribution strategy to control the motor driving torque or regenerative braking torque for vehicle stability enhancement [20]. Park et al. proposed a torque distribution strategy that considers the driving economics, driver's acceleration demand, and tire slip and used fuzzy logic to select a suitable distribution strategy [21]. Kim and Kim chose the regenerative braking and an electrohydraulic brake as the actuators in the vehicle stability controller. Aiming to minimize EHB energy consumption, the braking force distribution ratio of the front and rear axles were optimized [22].

According to the above studies, the hierarchical vehicle stability controller obtains the desired driving state by using the driver steering wheel angle. However, in-depth research showed that the use of only the steering wheel angle is inadequate to express the driver intention and obtain the desired driving state vehicle stability controller.
The driver turning intention is initiated based on experience depending on the condition of the road, environment, and vehicle state. Then, the driver manipulates the vehicle actuator such that the vehicle responds consistently with their intention. Therefore, the driver's turning intention reflects their subjective demand for the vehicle state. When drivers take sharp turning operation, such as go through continuous S-turn, hairpin bend, and the other roads with a smaller radius curvature, as well as emergency obstacle avoidance or sudden overtaking, the driver would be considered to intend rapidly maneuvering the vehicle under current road conditions, which also means the driver has certain expectations in terms of the vehicle yaw rate and sideslip. However, for some drivers, because of constraints such as their driving experience, the driving environment, theoretical knowledge, and other factors, this expectation ignores correct judgment of the lateral motion stability of the vehicle. During the process of vehicle stability control, if it is a significant difference between the actual and expected state of the vehicle, the driver would proceed with the turning operation, and the vehicle stability would deteriorate and even cause the driver to distrust the system.

Therefore, accurate interpretation and prediction of the driving intention during the process of vehicle stability control and ensuring the actual vehicle state is maintained as closely as possible to the expected vehicle state would greatly improve the stability and driving safety of the vehicle. Driver intention can be obtained using fuzzy reasoning, support vector machine (SVM), artificial neural network (ANN), and hidden Markov model (HMM) [23-27]. Fuzzy reasoning, SVM, and ANN are used to recognize the intentions of driver at a certain moment. However, neural network and fuzzy logic are difficult to deal with temporal-ordered information. Thus, they are mainly used for static recognition problems. HMM, as a kind of dynamic information processing method based on time-series cumulative probability, considers only the state sequence with the maximum loglikelihood and ignores the possibility of small probability events. Therefore, it is difficult to recognize easily confused intentions using HMM.

All the above analysis motivated us to develop a method to detect the driver's turning intention, establish a steering urgency coefficient for sharp turning, and propose a vehicle stability control reference model considering the driver's turning intention. Based on the abovementioned results, we proposed a stability control strategy for a dual-motor drive electric vehicle. As a result of this research, the desired control target of stability control is no longer only based on the steering wheel angle, and the vehicle state, in accordance with the driving intention, is under control to a certain extent. This paper is organized as follows. The method to identify the driver's turning intention is presented in Section 1. A modified reference model for vehicle stability considering the driver's turning intention is presented in Section 2. Construction of the vehicle stability control strategy based on the modified reference model is discussed in Section 3. Section 4 compares the vehicle stability control strategy based on the modified reference model constructed in this 
work with the traditional strategy. Finally, Section 5 presents the conclusions of this study.

\section{Driver's Turning Intention Recognition Hybrid Model of GHMM and GGAP-RBF Neural Network}

The turning operation is a complex event that continues for a certain period of time. The observation sequence of the turning process is a set of temporal data. The Gaussian hidden Markov model (GHMM) displays a strong modeling ability for dynamic time sequences. However, the model does not take into account overlaps between different classes, and this is a severe limitation. In contrast, generalized growing and pruning RBF (GGAP-RBF), an engineering model, can simulate the thinking mechanism of the human brain, has strong classification and decisionmaking abilities and can describe uncertain information. Thus, it compensates for the inadequacies of HMM. In addition, the model allows insignificant neurons to be removed in each iterative training cycle to effectively control the growth of the neural network and simplify the structure of a network with large data capacity. However, the ability of GGAP-RBF to describe dynamic sequential processes is not especially strong [28-30]. This led us to construct a hybrid model in the form of a GHMM/GGAPRBF neural network. Given the advantages of sequential model building and its nonlinear mapping ability, the hybrid model can obtain newly identified information, thereby considerably increasing the accuracy of the classification of classes with slight differences [27]. At the same time, to improve the real-time performance, this work uses the initial stage of the turning operation to identify the driver's turning intention.

The structure of the driver turning intention recognition system based on the GHMM/GGAP-RBF hybrid model is shown in Figure 1. The hybrid model includes a lower layer (the GHMM model) and an upper layer (the GGAP-RBF model). The lower layer of the model includes the sharp turning GHMM and the normal turning GHMM. The vehicle speed, the steering wheel angle, the steering wheel angle velocity, and the steering wheel torque are the inputs of the lower layer. The log-likelihood of the sharp turning GHMM and the normal turning GHMM are the outputs of the lower layer. Based on the data of the turning initial stage, the normal turning and sharp turning GHMMs are designed and trained using the Baum-Welch algorithm to calculate the most likely sequence of states. In addition, a forward algorithm is used to calculate the log-likelihood of the GHMMs.

In the upper layer, the log-likelihood of the GHMM, the vehicle yaw rate, and the lateral acceleration form a vector $\left\{\delta_{T}^{1}(1), \delta_{T}^{1}(2), \ldots, \delta_{T}^{1}(N)\right\}$. The nonlinear combination of this vector is regarded as the input of the GGAP-RBF neural network, and the nonlinear mapping ability of neural network-based methods is used to recognize the driver actual turning intention.

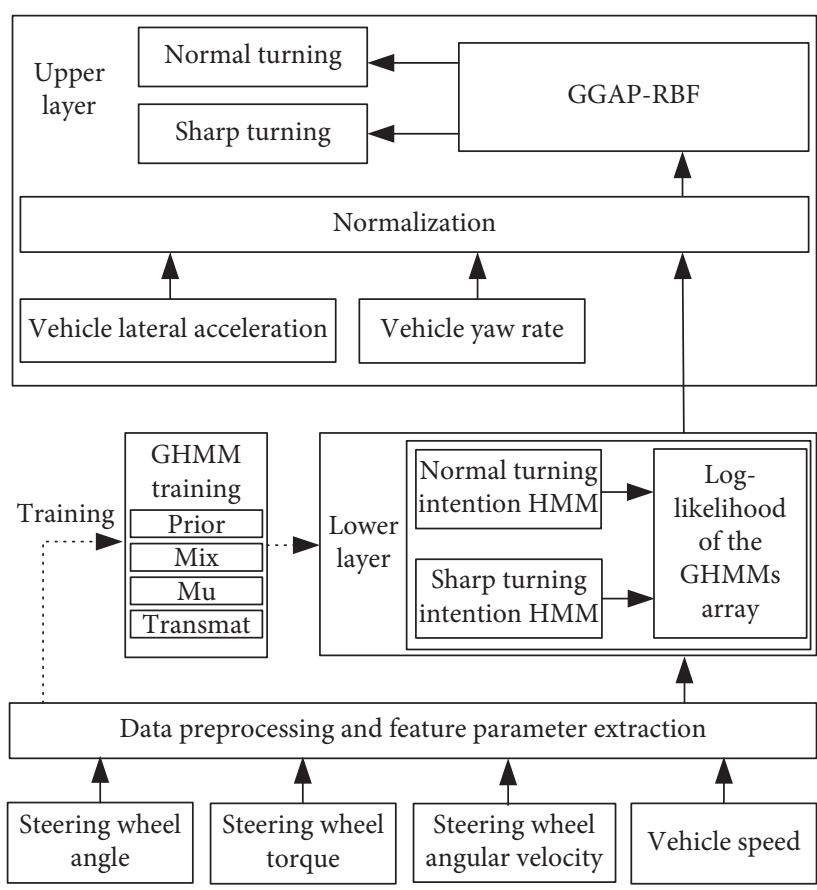

FIGURE 1: Structure of the GHMM/GGAP-RBF hybrid model.

2.1. Establishment of the GHMM. The feature parameters of the GHMM/GGAP-RBF hybrid model have a considerable effect on the accuracy of the recognition of the driver's turning intention. The reliefF algorithm is used to collect the appropriate parameters. This study employs the steering wheel angle, steering wheel angle velocity, and steering wheel torque as feature parameters to recognize the driver's steering intention. Based on the initial stage of the turning operation, sharp turning and normal turning GHMMs are established separately. The observation sequence of the GHMM can be described as a multidimensional vector [31]:

$$
O_{t}=\{a(t), b(t), c(t)\},
$$

where $a(t)$ is the steering wheel angle, $b(t)$ is the steering wheel torque, and $c(t)$ is the steering wheel angular velocity.

The Baum-Welch algorithm is used to optimize the three parameters of the GHMM, which are described as $\lambda=(\pi, A, B)$, where $\pi$ is the initial state distribution, $\mathrm{A}$ is the state transition probability matrix, and $B$ is a probability density function.

The probability density function of the model is

$$
b_{i}(O)=\sum_{j=1}^{M} \omega_{i j} N\left(O, \mu_{i j}, \sigma_{i j}\right),
$$

where $N\left(O, \mu_{i j}, \sigma_{i j}\right)$ is the $j$-dimensional Gauss probability density of state $i, O$ is the observation sequence, $\mu_{i j}$ is the mean of the Gauss function, and $\sigma_{i j}$ is the covariance of the Gauss function.

Assuming that $\varepsilon_{t}(i, j)$ is the probability of the $j$ th Gaussian mixture function in the state observation sequence $i$ at time $t$, the probability that the Markov chain is in state $j$ at time $t+1$ is as follows: 


$$
\begin{aligned}
\varepsilon_{t}(i, j) & =\frac{p\left(s_{t}=i, s_{t+1}=j, O \mid \lambda\right)}{p(O \mid \lambda)} \\
& =\frac{\alpha_{t}(i) \beta_{t}(i)}{\sum \alpha_{t}(i) \beta_{t}(i)} \times \frac{\omega_{i j} P\left[O, \mu_{i j}, \sigma\right]}{\sum_{1}^{K} \omega_{i j} P\left[O, \mu_{i j}, \sigma\right]},
\end{aligned}
$$

where $\mu_{i j}$ is the mean matrix of the Gaussian mixture function, $\sigma$ is the mixed covariance matrix, and $\omega_{i j}$ is the weight of output probabilities of different Gaussian mixture functions. Based on the Gaussian mixture model, the parameter re-estimation is as follows:

$$
\begin{aligned}
\omega_{i j}^{\prime} & =\frac{\sum_{t=1}^{T} \varepsilon_{t}(i, j)}{\sum_{t=1}^{T} \sum_{t=1}^{K} \varepsilon_{t}(i, j)} \\
\mu_{i j}^{\prime} & =\frac{\sum_{t=1}^{T} \varepsilon_{t}(i, j) O_{t}}{\sum_{t=1}^{T} \varepsilon_{t}(i, j)} \\
\sigma_{i j}^{\prime} & =\frac{\sum_{t=1}^{T} \varepsilon_{t}(i, j)\left(O_{t}-\mu_{i j}\right)\left(O_{t}-\mu_{i j}\right)^{\prime}}{\sum_{t=1}^{T} \varepsilon_{t}(i, j)} .
\end{aligned}
$$

After optimization of the parameters of the GHMM, the matching between the collected data and GHMM is calculated using the forward-backward algorithm.

\subsection{Establishment of the GGAP-RBF Neural Network. In} order to ensure that small probability events can also happen, function (5) is no longer used to recognize the turning intention. Instead, turning intention is described as a function of the log-likelihood of the two GHMMs given test data $(\log l i k 1$ and $\log l i k 2)$, the vehicle yaw rate $\omega_{\mathrm{r}}$, and the lateral acceleration $a_{y}$ expressed as follows:

$$
\begin{aligned}
& \text { inention }=\max (\operatorname{loglik} 1, \operatorname{loglik} 2), \\
& \text { inention }=F\left(\log \text { lik1, loglik } 2, \omega_{\mathrm{r}}, a_{y}\right) .
\end{aligned}
$$

The input parameter of the input layer is $\operatorname{loglik} 1^{*}$, $\operatorname{loglik} 2^{*}, \omega_{\mathrm{r}}^{*}$, and $a_{y}^{*}$, as shown in equation (7). The output of the layer is the driver turning intention, as shown in equation (8):

$$
\begin{gathered}
x_{i}=\left[\operatorname{loglik} 1^{*}, \operatorname{loglik} 2^{*}, \omega_{\mathrm{r}}^{*}, a_{y}^{*}\right], \\
f\left(x_{i}\right)=\operatorname{loglik}\left(x_{i}\right)=\omega_{0}+\sum_{k=1}^{K} \omega_{k} \cdot e^{-\left(\left\|w_{k}-x_{i}\right\|^{2} / \sigma_{k}^{2}\right)},
\end{gathered}
$$

where $\operatorname{loglik} 1^{*}, \log \mathrm{lik} 2^{*}, \omega_{\mathrm{r}}^{*}$, and $a_{y}^{*}$ represent the deviation standardization of $\log l i k 1, \log l i k 2, \omega_{\mathrm{r}}$, and $a_{y} ; \omega_{k}$ is the center of the RBF of the $k$ th neuron; and $\sigma_{k}$ is the standard deviation of the Gaussian function, which indicates the width of the Gaussian function.

After the $i$ th training iteration, RAN is used to optimize the growth of neurons. The parameters of a neuron, upon addition of a new neuron, are given as follows [32-35]:

$$
\left\{\begin{array}{l}
\omega_{\mathrm{n}}=e_{i}=y_{i}-f^{(i-1)}(x), \\
w_{\mathrm{n}}=x_{i}, \\
\sigma_{\mathrm{n}}=\kappa\left\|x_{i}-w_{i r}\right\|,
\end{array}\right.
$$

where $\omega_{\mathrm{n}}$ is the weight connecting the new neuron to the output neuron; $w_{\mathrm{n}}$ is the center of the new neuron; $\sigma_{\mathrm{n}}$ is the width of the new neuron; $e_{i}=y_{i}-f^{(i-1)}(x)$ is the error of the a priori estimate; $\kappa$ is an overlap factor that determines the overlap of the responses of the hidden neurons in the input space; and $w_{i r}$ is the value of the center of the hidden neuron nearest to $x_{i}$.

The worth of the new neuron is estimated using the conditions in inequality (10). If the conditions are met, the new training data are valuable to the network, and the network performance is improved effectively by the new neuron. As the result, the new neuron is added, and the training data are accepted. If not, the training data and the new neuron are rejected.

$$
\left\{\begin{array}{l}
\left\|x_{i}-w_{i r}\right\|>\varepsilon_{i}, \\
e_{i}=y_{i}-f\left(x_{i}\right)>e_{\min },
\end{array}\right.
$$

where $\varepsilon_{i}$ and $e_{\min }$ are the threshold of the distance and the error, respectively; $w_{i r}$ is the value of the center of the hidden neuron nearest to $x_{i}$; and $e_{i}$ is the error of the priori estimate.

Then, insignificant neuron should be judged and removed using a pruning algorithm. The mean squared error of prediction output after the $k$ th neuron is removed from the network in the $i$ th training iteration as follows:

$$
\begin{aligned}
E_{q}(k) & =\|\varepsilon(k, 1), \varepsilon(k, 1), \ldots, \varepsilon(k, n)\|_{q} \\
& =\left(\frac{1}{n} \sum_{i=1}^{n} \varepsilon^{q}(k, i)\right)^{1 / q}=\left\|\omega_{k}\right\|_{q}\left(\frac{1}{n} \sum_{i=1}^{n} R_{k}^{q}\left(x_{i}\right)\right)^{1 / q},
\end{aligned}
$$

where $R_{k}\left(x_{i}\right)$ is the Gaussian RBF, $n$ is the training time, \|\|$_{q}$ is the norm, and $k$ is the $k$ th hidden neuron.

The inputs of the RBF, $\log \operatorname{lik} 1^{*}, \log \operatorname{lik} 2^{*}, \omega_{\mathrm{r}}^{*}$, and $a_{y}^{*}$, follow the normal sampling distribution $N\left(\mu, \sigma^{2}\right)$, respectively. The sample range of the $i$ th training data $\left(x_{i}, y_{i}\right)$ is $X$, which is divided into $J$ equal small parts $\Delta_{j}$. As $\Delta_{j}$ tends to infinity, the sum over the sample range becomes approximately equal to the integral value.

$$
\begin{aligned}
E_{\text {sig }}(k) & =\lim _{J \longrightarrow \infty} E_{q}(k) \\
& =\left\|\omega_{k}\right\|_{q} \prod_{l=1}^{L}\left(\int_{a_{l}}^{b_{l}} e^{-\left(\left\|w_{k}-x_{i, l}\right\|^{2} / \sigma_{k}^{2}\right)} p_{l}(x) \mathrm{d} x\right)^{1 / q},
\end{aligned}
$$

where $E_{\text {sig }}(k)$ is the significance of the $k$ th neuron to the network, which means the contribution of that neuron to the entire network and $P_{1}(x)$ is the probability distribution function. If $E_{\text {sig }}(k)$ is less than the learning accuracy $e_{\text {min }}$, the neuron is considered to be insignificant and is removed. 
Otherwise, the neuron is significant and should be retained. Because insignificant neurons can be removed from the GGAP-RBF neural network, the size of the network can be limited to a reasonable range.

2.3. Establishment of GHMM/GGAP-RBF Model. The establishment of GHMM/GGAP-RBF model needs offline training by using test data for which is obtained in real vehicle experiment.

Driving experience, gender, and personality can affect driver's decision-making. In order to eliminate the influence of drivers on the test results, three drivers with different driving experiences are selected. According to the analysis of Specification for Design of Municipal Roads (CJJ37-2012), Specification for Design of Interactions on Urban Road (CJJ152-2010), and Vehicle Handling Stability Test Method (GB/T6323-2 2014), the radius of turning the test road is set as $10 \mathrm{~m}, 25 \mathrm{~m}, 40 \mathrm{~m}$, and $60 \mathrm{~m}$. The radius of turning the test road is set as $10 \mathrm{~m}, 25 \mathrm{~m}, 40 \mathrm{~m}$, and $60 \mathrm{~m}$. And, the tests speed is set as $20 \mathrm{~km} / \mathrm{h}, 30 \mathrm{~km} / \mathrm{h}$, and $40 \mathrm{~km} / \mathrm{h}$. For distinguishing between the straight driving and turning, straight driving tests are also conducted at $20 \mathrm{~km} / \mathrm{h}, 30 \mathrm{~km} / \mathrm{h}$, and $40 \mathrm{~km} / \mathrm{h}$. The distribution of test data is shown in Table 1.

The vehicle parameters are shown in Table 2. Because of the noise in the sensor data collected by the data acquisition instrument, the data must be preprocessed. T-testing is used to remove abnormal data. The mixed Gaussian clustering method is then used to extract data pertaining to the initial stage of the turning operation as part of the entire turning process. These preprocessed data can be divided into two parts. $75 \%$ of the test data is used for model offline training, and the other is used for model online verification. After the offline training, the initial state matrix, the state transition matrix, the weight of each Gaussian function in the GHMM, the mean and covariance of each Gaussian function, and the parameters of the GGAP-RBF could be gotten.

\section{Reference Model of Vehicle Stability Control System considering the Driver's Intention}

This paper proposes a reference model for vehicle stability control. The model, which takes the driver's turning intention into consideration, is shown in Figure 2. First, the GHMM/GGAP-RBF hybrid model is used to recognize the driver's turning intention on the basis of data relating to the steering wheel operation: angle, angular velocity, and steering wheel torque. When, at the initial stage of the turning operation, the driver's intention is identified as sharp turning, the vehicle is made to respond quickly to the desired yaw rate for the current steering wheel angle (i.e., the yaw rate can follow the driver's steering wheel operation successfully) by correcting the reference yaw rate with the aid of the established steering urgency coefficient. When the steering operation enters the turning keeping stage and the turning reversal stage, vehicle stability is ensured by no longer modifying the reference yaw rate. The
TABle 1: The distribution of test data.

\begin{tabular}{lccc}
\hline & $20 \mathrm{~km} / \mathrm{h}$ & $30 \mathrm{~km} / \mathrm{h}$ & $40 \mathrm{~km} / \mathrm{h}$ \\
\hline Sharp turning & 80 & 80 & 60 \\
Normal turning & 88 & 88 & 68 \\
Straight driving & 30 & 21 & 21 \\
\hline
\end{tabular}

TABLE 2: Vehicle parameters.

\begin{tabular}{lc}
\hline Parameter & Value \\
\hline Vehicle mass & $443.9 \mathrm{~kg}$ \\
Axle load distribution & $45: 55$ \\
Wheelbase & $1200 \mathrm{~mm}$ \\
Axle base & $1550 \mathrm{~mm}$ \\
Height of mass center & $281.9 \mathrm{~mm}$ \\
Distance from the center of mass to the front axis & $854.2 \mathrm{~mm}$ \\
Distance from the center of mass to the rear axis & $695.8 \mathrm{~mm}$ \\
Rated power $(\mathrm{kW})$ & 32 \\
Peak power $(\mathrm{kW})$ & 80 \\
Rated torque $(\mathrm{Nm})$ & 80 \\
Peak torque $(\mathrm{Nm})$ & 160 \\
\hline
\end{tabular}

reference model is not modified when normal turning is intended.

3.1. Reference Model Based on 2-DOF Linear Vehicle Dynamic Model. The most commonly used vehicle stability control reference model is the 2-DOF linear vehicle dynamic model, which only considers the lateral motion and yaw motion of the vehicle [36]. The state equations are as follows:

$$
\begin{aligned}
& m\left(\dot{v}-u \omega_{\mathrm{r}}\right)=\left(k_{1}+k_{2}\right) \beta \\
&+\frac{1}{u}\left(a k_{1}-b k_{2}\right) \omega_{\mathrm{r}}-k_{1} \delta_{\mathrm{f}}, \\
& I_{Z} \dot{\omega}_{\mathrm{r}}=\left(a k_{1}-b k_{2}\right) \beta+\frac{1}{u}\left(a^{2} k_{1}+b^{2} k_{2}\right) \omega_{\mathrm{r}}-a k_{1} \delta_{\mathrm{f}} .
\end{aligned}
$$

Thus, the ideal yaw rate and sideslip angle are as follows:

$$
\begin{gathered}
\omega_{\text {rdes }}=\frac{u}{R}=\frac{u \delta_{\mathrm{f}}}{a+b+\left(\left(m u^{2}\left(b k_{2}-a k_{1}\right)\right) / 2 k_{1} k_{2}(a+b)\right)}, \\
\beta_{\text {des }}=\frac{b-\left(a /\left(2(a+b) k_{2}\right)\right) m u^{2}}{a+b+\left(\left(m u^{2}\left(b k_{2}-a k_{1}\right)\right) / 2 k_{1} k_{2}(a+b)\right)} \delta_{\mathrm{f}} .
\end{gathered}
$$

When the vehicle is driving on a road with a low adhesion coefficient, e.g., when the road surface is wet or covered by snow or sand, the adhesion force allowed by the adhesion conditions between the road surface and tires decreases and cannot produce the high yaw rate required by the vehicle. Therefore, when the 2-DOF linear vehicle dynamic model is adopted as the ideal model, it must be limited by the conditions under which the tires adhere to the road.

The upper boundary of the ideal yaw rate is 


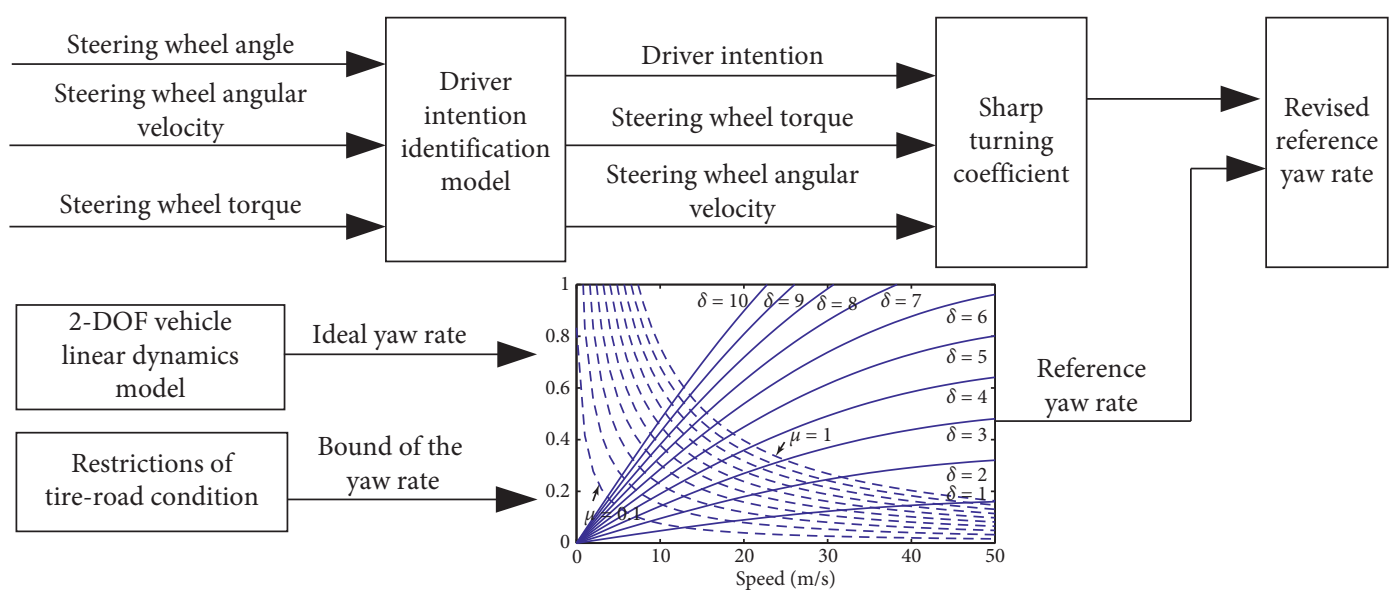

Figure 2: Modified model of reference yaw rate.

$$
\omega_{\text {rupper_bound }}=0.85 \frac{\mu g}{u} \text {. }
$$

Therefore, the reference value of the vehicle yaw rate for steady-state steering is

$$
\omega_{\text {rref }}= \begin{cases}\omega_{\text {rdes }}, & \left|\omega_{\text {rdes }}\right| \leq\left|\omega_{\text {rupper_bound }}\right|, \\ \omega_{\text {rupper_bound }} \operatorname{sgn}\left(\omega_{\text {rdes }}\right), & \left|\omega_{\text {rdes }}\right|>\left|\omega_{\text {rupper_bound }}\right| .\end{cases}
$$

Similarly, the upper bound of the ideal sideslip angle must be specified such that it is not too large. The upper boundary of the sideslip angle is

$$
\beta_{\text {upper_bound }}=\tan ^{-1}(0.02 \mu \mathrm{g}) \text {. }
$$

Therefore, the reference value of the sideslip angle for steady-state steering is

$$
\beta_{\text {ref }}= \begin{cases}\beta_{\text {des }}, & \left|\beta_{\text {des }}\right| \leq\left|\beta_{\text {upper_bound }}\right|, \\ \beta_{\text {upper_bound }} \operatorname{sgn}\left(\beta_{\text {des }}\right), & \left|\beta_{\text {des }}\right|>\left|\beta_{\text {upper_bound }}\right| .\end{cases}
$$

This enables the basic control target of the stability control system, namely, the reference yaw rate $\omega_{\text {rref }}$ and sideslip angle $\beta_{\text {ref }}$, to be obtained.

3.2. Reference Model Considering Driver's Intention. As mentioned in Section 1, for the same angle, angular velocity and torque of steering wheel can be used as characteristic parameters to recognize the driver's turning intention. Therefore, the steering wheel angular velocity and torque are taken as parameters to establish the steering urgency coefficient for sharp turning intention.

When the vehicle is undergoing steady-state turning, the relationship between the steering torque and the gradient of lateral acceleration satisfies [37]

$$
\frac{\mathrm{d} M_{\mathrm{L}}}{\mathrm{d}\left(v^{2} / \rho\right)}=\frac{m n_{v} l_{H}}{i_{\mathrm{L}} V_{\mathrm{L}} l}=\frac{1}{g} \frac{1}{i_{\mathrm{L}} V_{\mathrm{L}}} F_{z v} n_{v}
$$

Therefore, according to the actual lateral acceleration, the ideal steering wheel torque for steady-state turning can be calculated as

$$
M_{\text {Ldes }}=\int \frac{1}{g} \frac{1}{i_{\mathrm{L}} V_{\mathrm{L}}} F_{z v} n_{v} \mathrm{~d}\left(\frac{v^{2}}{\rho}\right) .
$$

According to equation (21) and the model of the steering system, the actual yaw rate and speed can be used to calculate the ideal steering wheel angular velocity by differentiating

$$
\left\{\begin{array}{l}
\delta_{\text {wdes }}=\frac{L\left(1+K u^{2}\right)}{u} \omega_{\mathrm{r}}, \\
K=\frac{m}{L^{2}}\left(\frac{a}{k_{2}}-\frac{b}{k_{1}}\right) . \\
\delta_{\text {swdes }}=f\left(\delta_{\text {wdes }}\right) .
\end{array}\right.
$$

The deviation between the actual torque and angular velocity and the ideal torque and angular velocity of the steering wheel is used to reflect the urgency of the driver's sharp turning intention. Finally, the steering urgency coefficient for sharp turning intention is calculated by the following equation:

$$
\tau=\frac{1}{2} \sqrt{\left(\bar{\delta}_{\text {sw }}-\bar{\delta}_{\text {swdes }}\right)^{2}+\left(\bar{M}_{\mathrm{L}}-\bar{M}_{\text {Ldes }}\right)^{2}}
$$

where $\bar{\delta}_{\text {sw }}, \bar{\delta}_{\text {swdes }}, \bar{M}_{\mathrm{L}}$, and $\bar{M}_{\text {Ldes }}$ is the normalized actual steering wheel torque, actual angular velocity, ideal steering wheel torque, and ideal angular velocity using the following equation to avoid the impact caused by the different dimensions of each parameter.

$$
\bar{x}=\frac{x-\min }{\max -\min } .
$$

When $\left|\omega_{\text {rdes }}\right| \leq\left|\omega_{\mathrm{r}}\right| \leq\left|\omega_{\text {rupper_bound }}\right|$, the reference yaw rate is determined by the dynamic characteristics of the vehicle, and the limitation generated by the adhesion condition of the road is not reached. If the driver intention is sharp turning at the same time, the reference yaw rate would be modified as follows: 


$$
\omega_{\text {rref }}=\omega_{\text {rdes }}+\Delta \omega_{\mathrm{r}}
$$

where $\Delta \omega_{\mathrm{r}}=\tau\left(\omega_{\mathrm{r}}-\omega_{\text {rdes }}\right)$ is the correction of the reference yaw rate considering the driver turning intention and is related to the urgency of the driver turning intention.

When $\left|\omega_{\text {rdes }}\right| \leq\left|\omega_{\text {rupper_bound }}\right| \leq\left|\omega_{\text {r }}\right|$, the reference yaw rate is determined by the dynamic characteristics of the vehicle, and the limitation generated by the adhesion condition of the road is also reached. If equation (25) is still adopted for modification, the reference yaw rate may exceed the limitation. Thus, under this condition, the reference yaw rate would be modified as follows:

$$
\Delta \omega_{\mathrm{r}}=\tau\left(\omega_{\text {rupper_bound }}-\omega_{\text {rdes }}\right) .
$$

When $\left|\omega_{\text {rupper_bound }}\right| \leq\left|\omega_{\text {rdes }}\right|$, the reference yaw rate is determined by the adhesion condition of the road. In order to ensure the driving safety, the reference yaw rate is no longer modified.

$$
\omega_{\text {rref }}^{*}=\left\{\begin{array}{l}
\omega_{\text {rdes }}+\tau\left(\omega_{\text {r }}-\omega_{\text {rdes }}\right), \\
\omega_{\text {rdes }}+\tau\left(\omega_{\text {rupper_bound }}-\omega_{\text {rdes }}\right), \\
\omega_{\text {rref }},
\end{array}\right.
$$

When the turning operation reaches the second stage-keeping stage and the third stage-returning stage, the reference yaw rate is no longer modified for the sake of the driving safety. The conversion from the modified model to the reference model causes the reference yaw rate to change suddenly, and this is most likely to influence the stability of the vehicle. In order to make the reference yaw rate smoother, the S-shaped acceleration and deceleration curve is chosen as the transition function, which is shown in Figure 3 [38].

When the inequality

$$
\omega_{\text {rref }}^{*}-\omega_{\text {rref }}>\varepsilon,
$$

is satisfied, the modified reference yaw rate is

$$
\omega_{\mathrm{rref}}^{*}(t)= \begin{cases}\omega_{\mathrm{rref}}^{*}\left(t_{0}\right)+\frac{1}{2} J t^{2}, & t_{0} \leq t \leq t_{1}, \\ \omega_{\mathrm{rref}}^{*}\left(t_{0}\right)-J t_{1}^{2}+2 J t_{1} t-\frac{1}{2} J t^{2}, & t_{1}<t \leq t_{2} .\end{cases}
$$

The flow of the calculation to obtain the reference yaw rate is shown in Figure 4.

\section{Vehicle Stability Control Strategy}

The proposed stability controller for dual-motor drive electric vehicle adopts a hierarchical structure, which includes the upper layer controller-with a decision layer for the additional yaw moment-and the lower layer controller-with a distribution layer for the additional yaw moment. The structure of this system is shown in Figure 5.

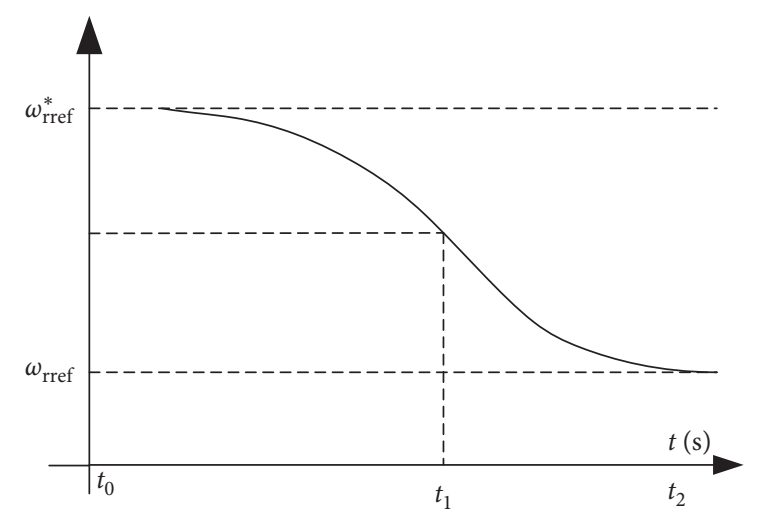

FIGURE 3: Switching transition function of reference yaw rate.

Thus, for the sharp turning intention, the reference yaw rate is

$$
\begin{aligned}
& \text { when }\left|\omega_{\text {rdes }}\right| \leq\left|\omega_{\mathrm{r}}\right| \leq\left|\omega_{\text {rupper_bound }}\right| \text {, } \\
& \text { when }\left|\omega_{\text {rdes }}\right| \leq\left|\omega_{\text {rupper_bound }}\right| \leq\left|\omega_{\mathrm{r}}\right| \text {, } \\
& \text { when }\left|\omega_{\text {rupper_bound }}\right| \leq\left|\omega_{\text {rdes }}\right| .
\end{aligned}
$$

The upper layer controller includes the vehicle state estimator model, the driver turning intention recognition model, the modified stability control reference model and the additional yaw moment decision model. This controller chooses the angle, angular velocity, and torque of the steering wheel as inputs to recognize the driver's turning intention. The reference yaw rate and reference sideslip angle under steady-state steering are determined by using the 2DOF linear vehicle dynamics model and by simultaneously considering the adhesion conditions. According to the turning intention, the reference model is modified as the final stability control target. Then, based on the difference between the actual and the reference yaw rate and sideslip angle, using the additional yaw moment decision model, the additional yaw moment that needs to be applied to restore the vehicle to the stable state is determined and used as input for the lower controller.

The lower layer controller-the additional yaw moment distribution layer-includes the longitudinal force distribution model and the actuator model. The generation of additional yaw moment requires the longitudinal force of the tire to be controlled, and this should take into consideration the vehicle drive form, performance of the motor and hydraulic brake system, and the road conditions. The optimization algorithm allocates additional yaw moment to get additional torque of the motor and hydraulic braking system which will eventually improve the stability of the vehicle.

4.1. Design of Upper Controller for Vehicle Stability Control. MPC is used to make decision of additional yaw moments. And, linear 3-DoF vehicle dynamics model including 


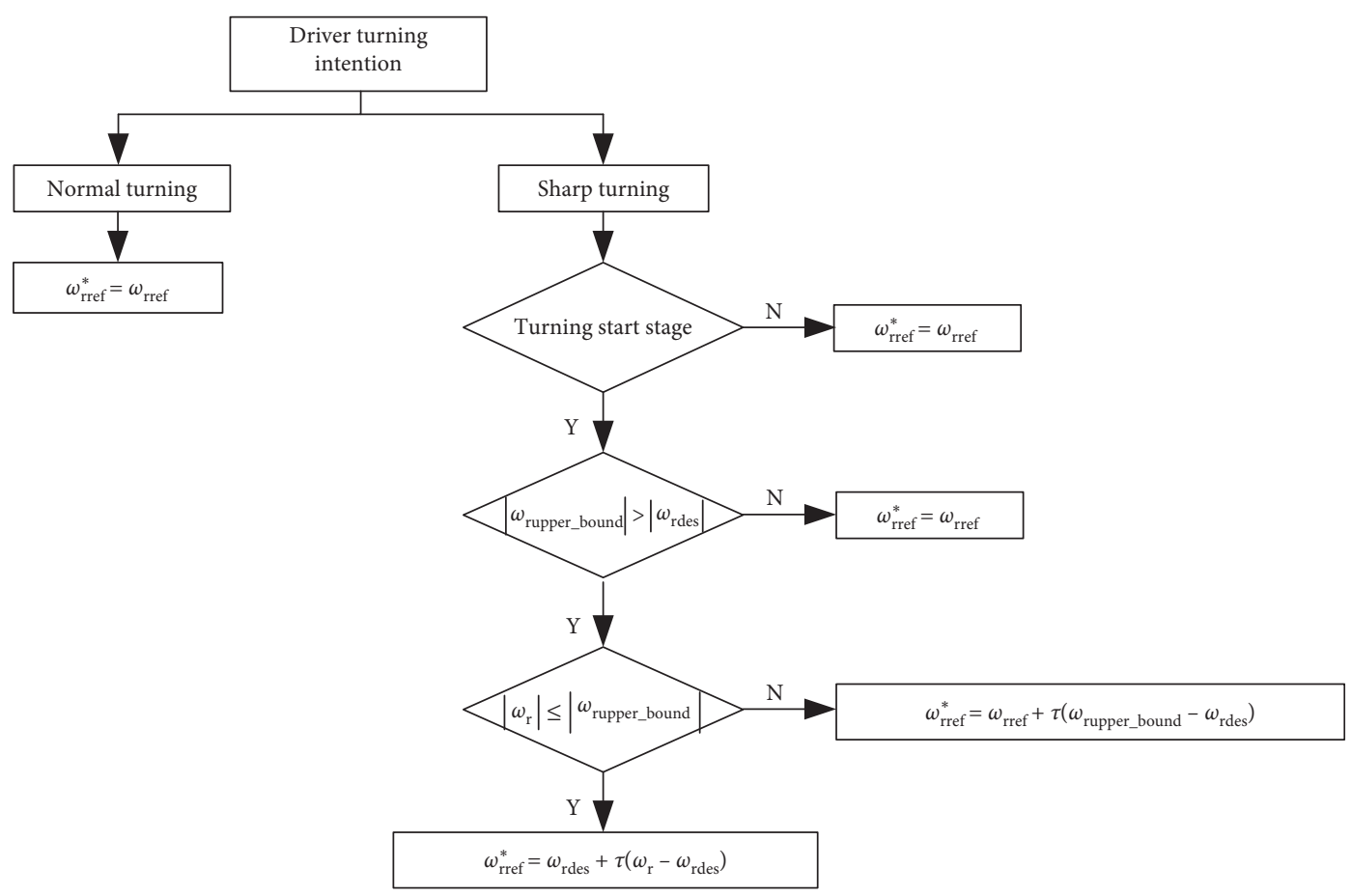

FIgURE 4: Flowchart of reference yaw rate.

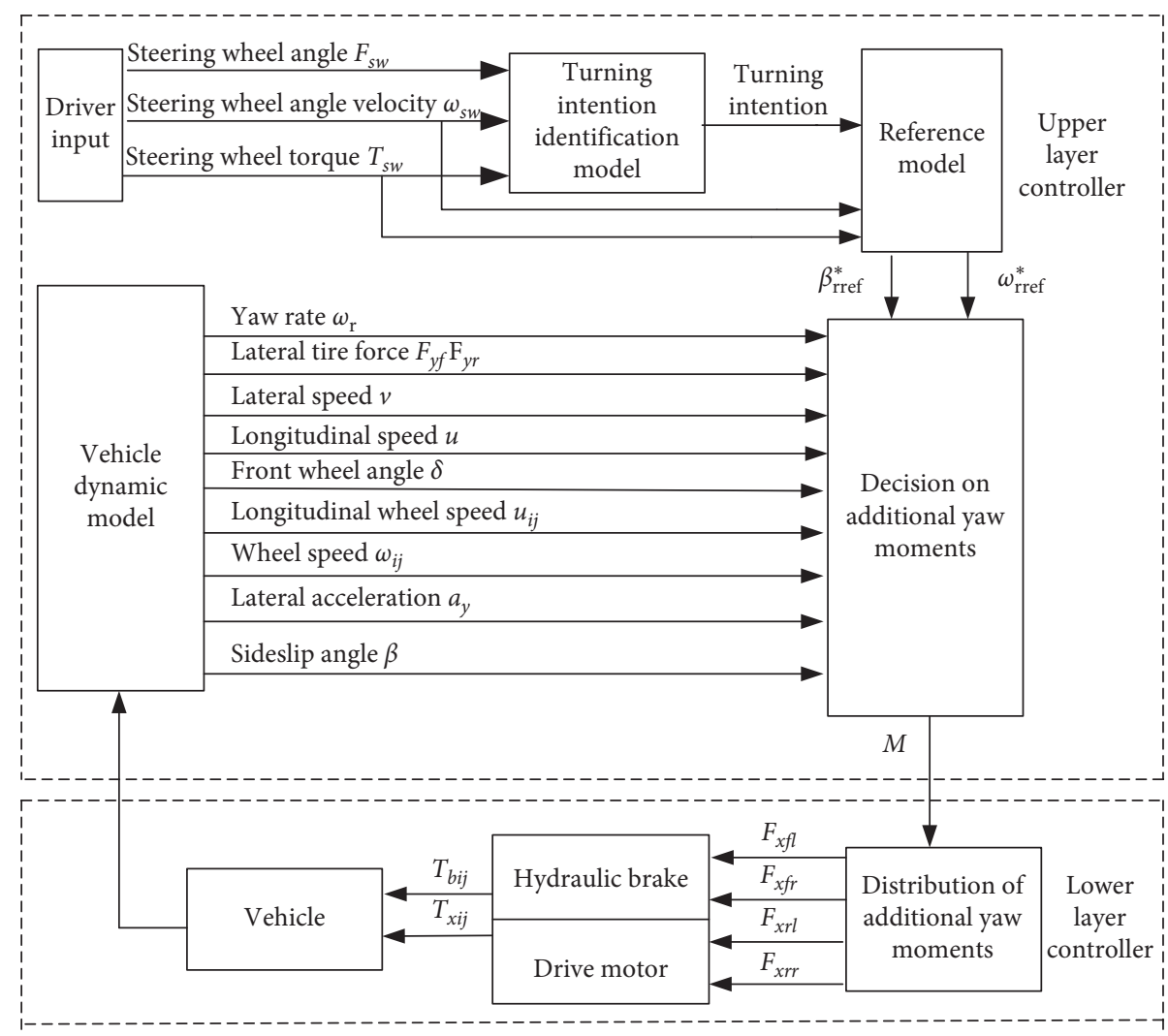

FIGURE 5: Structure of stability control system for dual-motor drive electric vehicle.

longitudinal motion, lateral motion, and yaw motion is selected as the predictive model, which is shown in Figure 6. Taking the yaw rate and the sideslip angle as the state variables, the vehicle state can be obtained as shown in equation (30)-(32) [39, 40]. For both accuracy and efficiency, the tire cornering stiffness estimator based on 


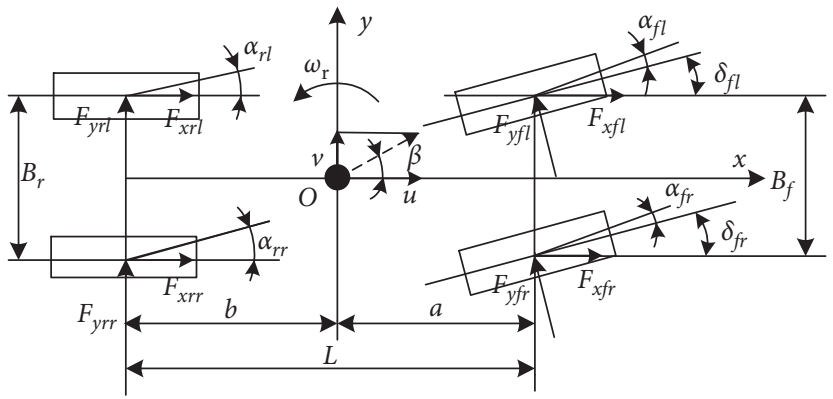

Figure 6: 3-DOF linear vehicle dynamics model.

recursive least square method with forgetting factor (FFRLS) is used to estimate the tire stiffness which will be used for calculating the tire lateral force in real time. Then, the estimated tire cornering stiffness is applied to the linear dynamic model. This improves the effectiveness of the control system in the nonlinear region of the tire [41].

$$
\begin{aligned}
\dot{V}= & \frac{\cos \beta}{m}\left(\cos \delta_{f l} F_{x r l}-\sin \delta_{f l} F_{y f l}+\cos \delta_{f r} F_{x f r}\right. \\
& \left.-\sin \delta_{f r} F_{y f r}+F_{x r l}+F_{x r r}\right)+\frac{\sin \beta}{m}\left(\sin \delta_{f l} F_{x r l}\right. \\
& \left.+\cos \delta_{f l} F_{y f l}+\sin \delta_{f r} F_{x f r}+\cos \delta_{f r} F_{y f r}+F_{y r r}+F_{y r l}\right),
\end{aligned}
$$

$\omega_{r}=\frac{1}{I_{z}}\left[\left(\cos \delta_{f r} F_{x r l}-\cos \delta_{f l} F_{x f l}\right) \frac{B_{f}}{2}+\left(F_{x r r}-F_{x r l}\right) \frac{B_{r}}{2}\right.$

$$
\begin{aligned}
& +a \sin \delta_{f r} F_{x r l}+b \sin \delta_{f l} F_{x f l} \\
& +\left(\frac{B_{f}}{2} \sin \delta_{f l}+a \cos \delta_{f l}\right) F_{y f l} \\
& \left.+\left(-\frac{B_{f}}{2} \sin \delta_{f r}+a \cos \delta_{f r}\right) F_{y f r}+a F_{y r l}+b F_{y r r}\right],
\end{aligned}
$$

$$
\begin{aligned}
\dot{\beta}= & \frac{\cos \beta}{m V}\left(\sin \delta_{f l} F_{x r l}+\cos \delta_{f l} F_{y f l}+\sin \delta_{f r} F_{x f r}\right. \\
& \left.+\cos \delta_{f r} F_{y f r}+F_{y r l}+F_{y r r}\right)-\frac{\sin \beta}{m V}\left(\cos \delta_{f l} F_{x r l}\right. \\
& \left.-\sin \delta_{f l} F_{y f l}+\cos \delta_{f r} F_{x f r}-\sin \delta_{f r} F_{y f r}+F_{x r r}+F_{x r l}\right) \\
& \cdot F_{y f l}-\omega_{\mathrm{r}} .
\end{aligned}
$$
follows:

The state equation can be expressed in standard form as

$$
\left\{\begin{array}{l}
\dot{X}=A_{c} X+B_{u c} U+B_{d c} D \\
Y_{c}=C_{c} X
\end{array}\right.
$$

where $X=\left[\omega_{\mathrm{r}} ; \beta ; V\right], Y_{c}=\left[\omega_{r} ; \beta\right], C_{c}=[1,0,0 ; 0,1,0], U$ is the additional yaw moment, and $D=\delta_{\mathrm{f}}$.

Equation (33) is discretized and converted into incremental form to obtain

$$
\left\{\begin{array}{l}
\Delta X(k+1)=A \Delta X(k)+B_{u} \Delta U(k)+B_{d} \Delta D(k), \\
Y(k)=C \Delta X(k)+Y(k-1),
\end{array}\right.
$$

where $\Delta X(k)=X(k)-X(k-1), \Delta U(k)=U(k)-U(k-1)$, $\Delta D(k)=D(k)-D(k-1), \quad A=e^{A_{c} T}, \quad B_{u}=\int_{0}^{T} e^{A_{c} \tau} B_{u c} \mathrm{~d} \tau$, $C=[1,0 ; 0,1]$, and $T$ is the control period.

The objective of vehicle stability control is to enable the actual vehicle yaw rate and sideslip angle to follow the reference value through the action of additional yaw moment. Therefore, at time $k$, the MPC optimization problem based on the linear model can be described as

$$
\begin{aligned}
\min J\left(Y(k), U_{k}\right)= & \left\|\bar{Y}(k)-Y_{\text {ref }}(k)\right\|_{\mathrm{Q}}^{2}+\|\Delta U(k)\|_{R}^{2} \\
= & \sum_{i}^{N_{\mathrm{p}}}\left\|\bar{Y}(k+i \mid k)-Y_{\text {ref }}(k+i \mid k)\right\|_{\mathrm{Q}}^{2} \\
& +\sum_{i}^{N_{c}-1}\|\Delta U(k+i \mid k)\|_{R}^{2} .
\end{aligned}
$$

The constraints of control variables, the increment of variables, and output of the model are as follows:

$$
\left\{\begin{array}{l}
U_{\min } \leq U(k) \leq U_{\max }, \\
\Delta U_{\min } \leq \Delta U(k) \leq \Delta U_{\max }, \\
Y_{\min } \leq Y(k) \leq Y_{\max } .
\end{array}\right.
$$

In this model, the predictive horizon $N_{\mathrm{p}}=5$, the control horizon $N_{\mathrm{c}}=3$, and the reference of the predictive model is $Y_{\text {ref }}=\left[\omega_{\text {rref }}^{*}, \beta_{\text {ref }}^{*}\right]$.

The desired control performance can be adjusted by using the weight matrix $Q$ and $R$, where $Q$ reflects the accuracy requirements of the system and $R$ reflects the size of the action required by the controller.

In this research,

$$
\begin{aligned}
& Q=\left[\begin{array}{cc}
10 & 0 \\
0 & 15
\end{array}\right], \\
& R=\left[\begin{array}{ccc}
500 & 0 & 0 \\
0 & 500 & 0 \\
0 & 0 & 8000
\end{array}\right] .
\end{aligned}
$$


As shown in equation (38), $\Delta U(k)$ is the increment of the sequence of control inputs, which is obtained by using the optimization objective and constraints during the sampling time of $k$, and $\bar{Y}(k)$ is the control output sequence which is obtained from the prediction model.

$$
\begin{array}{rl}
\bar{Y}(k) & =\left[\begin{array}{c}
Y(k+1 \mid k) \\
Y(k+2 \mid k) \\
Y(k+3 \mid k) \\
Y(k+4 \mid k) \\
Y(k+5 \mid k)
\end{array}\right] \\
\Delta U(k) & =\left[\begin{array}{c}
\Delta M(k \mid k) \\
\Delta M(k+1 \mid k) \\
\Delta M(k+2 \mid k)
\end{array}\right], \\
Y(k+1 \mid k) & C A x(k)+C B \Delta U(k), \\
Y(k+2 \mid k) & =C A^{2} x(k)+C A B \Delta U(k)+C B \Delta U(k+1), \\
\vdots & \\
Y(k+5 \mid k) & \sum_{i=1}^{5} C A^{i} x(k)+\sum_{i=1}^{5} C A^{i-1} B \Delta U(k) \\
+\cdots+\sum_{i=1}^{3} & C A^{i-1} B \Delta U(k+4)+\sum_{i=1}^{3} C A^{i-1} B \Delta \mathrm{d}(k)+Y(k) .
\end{array}
$$

Finally, only the first element of the optimized solution is applied to the system. Thus, the additional yaw moment is expressed by the following equation:

$$
M(k)=M(k-1)+\Delta M(k-1 \mid k) \text {. }
$$

4.2. Design of the Lower Controller for Vehicle Stability Control. The lower layer controller distributes the longitudinal forces of each wheel according to the output of additional yaw moment decision layer. The distribution is restricted by the adhesion condition between the tire and the road. Excessive additional longitudinal force, which causes longitudinal slip and further deteriorates the vehicle stability, should be avoided. This force is restricted by the performance of the motor and braking system. Excessive additional torque causes the motor and mechanical braking system to overload. The torque is also restricted by the working state of the motor; therefore, a state of system failure, in which the motor cannot provide braking force, should be avoided. Under the above constraints, the distributed longitudinal force needs to meet the demand of additional yaw moment.

4.2.1. Optimization Objective. The longitudinal and lateral forces, the tire can provide, are limited by the vertical load. As the longitudinal force of each tire should be distributed according to the vertical load, wheels with higher adhesion would be expected to play a greater role. Therefore, the optimization objective is to minimize the sum of the square of operating working load rate of each tire. Because of the limitation imposed by practical conditions, the lateral force of wheels cannot be directly controlled. In this study, the longitudinal force of tires is controlled to generate additional yaw moment. Thus, the optimization objective is expressed by the following equation:

$$
\begin{aligned}
\min J= & C_{f l} \frac{\left(F_{x f l}+\Delta F_{x f l}\right)^{2}}{\left(\mu_{f l} F_{z f l}\right)^{2}}+C_{f r} \frac{\left(F_{x f r}+\Delta F_{x f r}\right)^{2}}{\left(\mu_{f r} F_{z f r}\right)^{2}} \\
& +C_{r l} \frac{\left(F_{x r l}+\Delta F_{x r l}\right)^{2}}{\left(\mu_{r l} F_{z r l}\right)^{2}}+C_{r r} \frac{\left(F_{x r r}+\Delta F_{x r r}\right)^{2}}{\left(\mu_{r r} F_{z r r}\right)^{2}} .
\end{aligned}
$$

\subsubsection{Optimization Constraints}

(1) Equality Constraints. The strategy for distributing additional yaw moment should not only minimize the sum of the operating working load rate but also ensure that the longitudinal force meets the requirements of braking and acceleration operations of the driver and that the additional yaw moment meets the requirements of the upper controller.

$$
\begin{aligned}
m a_{x q}= & \left(F_{x f l}+\Delta F_{x f l}\right) \cos \delta_{w f l}+\left(F_{x f r}+\Delta F_{x f r}\right) \cos \delta_{w f r} \\
& +F_{x r l}+\Delta F_{x r l}+F_{x r r}+\Delta F_{x r r}, \\
\Delta M= & \frac{\Delta F_{x f l} \cos \delta_{w f l}}{2}-\frac{\Delta F_{x f r} \cos \delta_{w f r}}{2}+\frac{\Delta F_{x r l}}{2}-\frac{\Delta F_{x r r}}{2} .
\end{aligned}
$$

\section{(2) Inequality Constraint.}

(1) Adhesion condition constraint: when additional yaw moment is distributed, it is necessary to ensure that the longitudinal and lateral forces of the tire are within the tire adhesion ellipse to avoid longitudinal and lateral vehicle slip.

$$
\sqrt{F_{x i j}^{2}+F_{y i j}^{2}} \leq \mu_{i j} F_{z i j}
$$

(2) Motor and brake system performance constraints: the additional longitudinal force is also limited by the performance of the actuators. The additional longitudinal force of the two rear driving wheels is limited by the performance of the driving motor. The two nondriving front wheels can only provide braking force; thus, the additional longitudinal force of the four wheels is limited by the performance of the mechanical braking system.

The braking torque generated by motor is limited by the characteristics of the motor, which cannot exceed the maximum torque limit determined by the power generated at the current speed. The large dynamic fluctuation of the braking torque of the motor and the inverse proportion 
between the maximum braking torque and speed prevent the motor from generating an adequate amount of reverse electromotive force, such that the braking force generated by the motor is ineffective. Due to the large dynamic fluctuation of motor braking torque and the inverse proportion between the maximum braking torque and speed, the reverse electromotive force generated by the motor is too little when the motor is at low speed. The motor cannot generate effective braking force. Therefore, $500 \mathrm{r} / \mathrm{min}$ is set as the speed threshold. When the speed is lower than threshold, the system no longer uses motor braking. Therefore, the motor braking torque is required meet the limitation provided in the following equation: [42].

$$
T_{d \max }= \begin{cases}0, & 0<n \leq 500, \\ T_{\mathrm{e}}, & 500<n \leq n_{N}, \\ \frac{9550 P_{\mathrm{e}} / \eta}{n}, & n_{N}<n \leq n_{\max } .\end{cases}
$$

At the same time, control signal failure, power converter failure, insulation failure, and other types of failures of the motor may occur due to the design defects, the service environment and service life of the motor. Thus, the driving motor is no longer suitable to provide longitudinal force, so the motor failure coefficient $\tau_{i j}$ is introduced.

$$
\tau_{i j}= \begin{cases}0, & \text { motor is failed, } \\ 1, & \text { motor is working properly. }\end{cases}
$$

Therefore, considering the above factors comprehensively, additional longitudinal force of wheels would have to meet the constraints.

$$
\left\{\begin{array}{l}
\frac{T_{b f l \mathrm{max}}}{r}-F_{x f l} \leq \Delta F_{x f l} \leq-F_{x f l}, \\
\frac{T_{b f r \max }}{r}-F_{x f r} \leq \Delta F_{x f r} \leq-F_{x f r}, \\
\frac{T_{b r l \max }}{r}+\frac{T_{d \max } i \tau_{r l}}{r-F_{x r l}} \leq \Delta F_{x r l} \leq \frac{T_{d \max } i \tau_{r l}}{r-F_{x r l}}, \\
\frac{T_{b r r \max }}{r}+\frac{T_{d \max } i \tau_{r r}}{r-F_{x r r}} \leq \Delta F_{x r r} \leq \frac{T_{d \max } i \tau_{r r}}{r-F_{x r r}} .
\end{array}\right.
$$

Therefore, the optimal distribution of additional yawing moment can be expressed as follows:

$$
\begin{aligned}
& \int \min J\left(\Delta F_{x f l}, \Delta F_{x f r}, \Delta F_{x r l}, \Delta F_{x r r}\right)=C_{f l} \frac{\left(F_{x f l}+\Delta F_{x f l}\right)^{2}}{\left(\mu_{f l} F_{z f l}\right)^{2}}+C_{f r} \frac{\left(F_{x f r}+\Delta F_{x f r}\right)^{2}}{\left(\mu_{f r} F_{z f r}\right)^{2}}+C_{r l} \frac{\left(F_{x r l}+\Delta F_{x r l}\right)^{2}}{\left(\mu_{r l} F_{z r l}\right)^{2}}+C_{r r} \frac{\left(F_{x r r}+\Delta F_{x r r}\right)^{2}}{\left(\mu_{r r} F_{z r r}\right)^{2}} \\
& \text { s.t. }\left(F_{x f l}+\Delta F_{x f l}\right) \cos \delta_{w f l}+\left(F_{x f r}+\Delta F_{x f r}\right) \cos \delta_{w f r}+F_{x r l}+\Delta F_{x r l}+F_{x r r}+\Delta F_{x r r}=m a_{x q} \\
& \frac{\Delta F_{x f l} \cos \delta_{w f l}}{2}-\frac{\Delta F_{x f r} \cos \delta_{w f r}}{2}+\frac{\Delta F_{x r l}}{2}-\frac{\Delta F_{x r r}}{2}=\Delta M \\
& \sqrt{F_{x i j}^{2}+F_{y i j}^{2}} \leq \mu_{i j} F_{z i j} \quad(i j=f l, f r, r l, r r) \\
& \frac{T_{b f l \max }}{r}-F_{x f l} \leq \Delta F_{x f l} \leq-F_{x f l} \\
& \frac{T_{b f r \max }}{r}-F_{x f r} \leq \Delta F_{x f r} \leq-F_{x f r} \\
& \frac{T_{b r l \max }}{r}-F_{x r l} \leq \Delta F_{x r l} \leq \frac{T_{d \max } i}{r-F_{x r l}} \\
& \frac{T_{b r r \max }}{r}-F_{x r r} \leq \Delta F_{x r r} \leq \frac{T_{d \max } i}{r-F_{x r r}}
\end{aligned}
$$

\section{Test Verification}

Considering the practical difficulties associated with controller development, i.e., a long development cycle and high cost, we built a test platform for a dual-motor drive electric vehicle based on the A\&D5435 semiphysical simulation system and rapid prototyping technology. The tests were carried out under both double-lane and single-lane change 


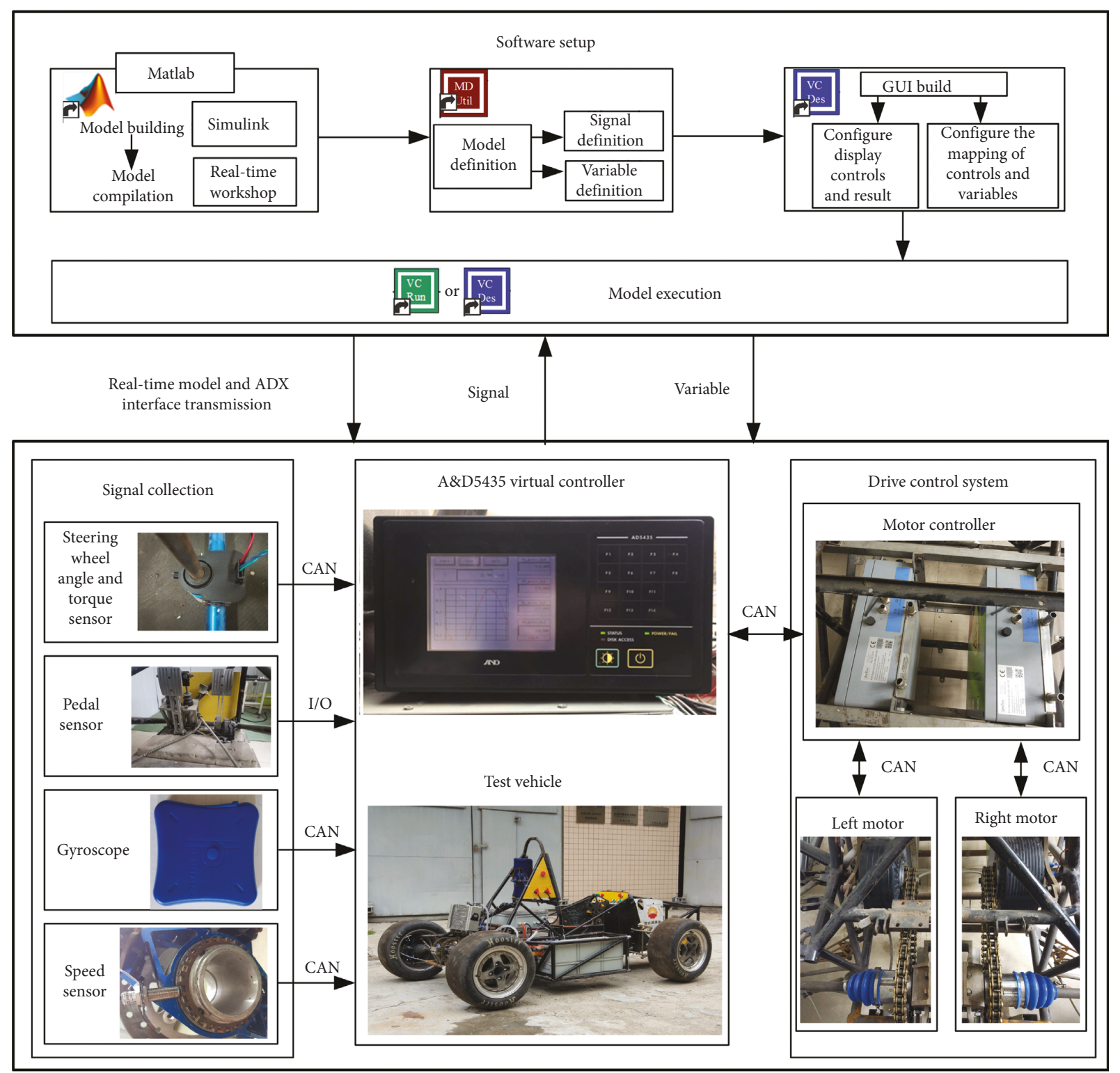

Figure 7: The dual-motor drive electric vehicle test platform.

conditions, respectively, to verify the feasibility and accuracy of the proposed stability control strategy.

5.1. Dual-Motor Drive EV Test Platform Based on the A\&D5435 Semiphysical Simulation System. In this study, a dual-motor drive electric vehicle test platform was built based on the A\&D5435 hardware in the loop simulation system and a dual-motor drive electric test vehicle. The stability control system proposed in this paper was tested and verified by using this platform in which the A\&D5435 replaces the vehicle control unit. The input signals of the controller include: angle, angular velocity and torque of the steering wheel, accelerator pedal opening, brake pedal opening, vehicle speed, wheel speed, motor torque, and motor power. The output signals include the following: the motor drive torque and the brake torque of both the motor and the hydraulic braking system. The input signal

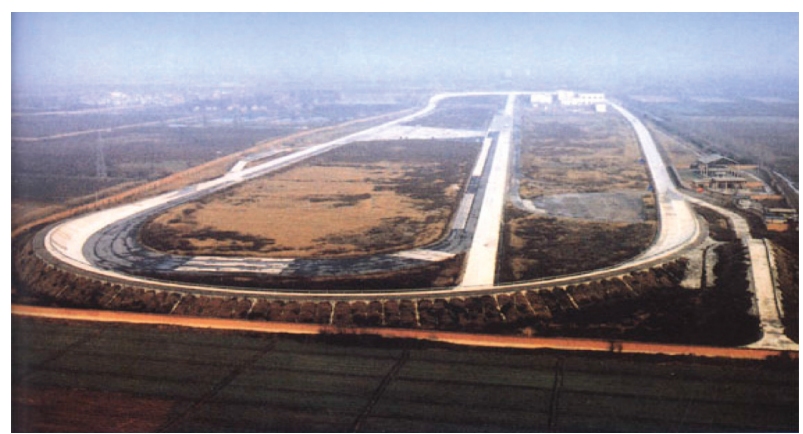

FIgURE 8: Chang'an University vehicle comprehensive performance proving ground.

mentioned above can be obtained by using the following sensors. The angle, angular velocity, and torque of the steering wheel can be collected using the steering wheel 


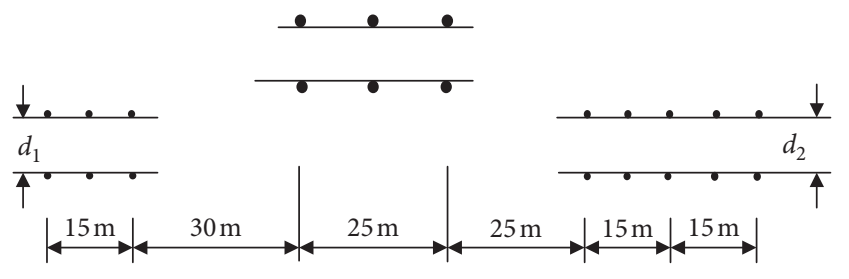

Figure 9: Schematic diagram for double-lane change test $\left(d_{1}=1.1 L+0.25 ; d_{2}=1.3 L+0.25 ; L\right.$ is the vehicle width $)$.

torque and angle sensor manufactured by SensorWay, which is mounted on the steering column. A hall noncontact speed sensor is used to record the speed of the four wheels. A Passat B5 accelerator pedal sensor, which has two channels, is used to measure the accelerator pedal opening. Likewise, the brake pedal opening is measured by the brake pedal sensor. The longitudinal acceleration, lateral acceleration, and yaw rate of the vehicle are acquired by a three-axis gyroscope. The motor speed, torque, and power are obtained from the CAN signal of the motor controller. The vehicle parameters are shown in Table 2. The dual-motor drive electric vehicle test platform is shown in Figure 7.

5.2. Test Verification: Road Tests. The stability control strategy of dual-motor drive EV proposed in this paper was verified by carrying out vehicle road tests involving both double-lane and single-lane change, respectively. At the same time, in order to demonstrate the effect of the proposed control strategy, the slide mode control (SMC) with the vehicle reference model considering the driver's intention is chosen as the comparative controller.

The vehicle road test was conducted on the stability performance test square of the Chang'an University vehicle comprehensive performance proving ground, as shown in Figure 8.

5.2.1. Double-Lane Change Condition. The test was conducted under ISO 3888-1 which specifies the standard double-lane change condition [43]. The target driving path is shown in Figure 9. The adhesion coefficient of the test road was 0.6 .

First, the driver was required to keep the vehicle parallel with the road and keep the steering wheel facing forward. Subsequently, the driver controlled the vehicle to rapidly proceed ahead, such that the vehicle speed before entering the target path reached $70 \mathrm{~km} / \mathrm{h}$, during which time the steering wheel was not to be operated. The driver could then drive freely by negotiating the cone track.

The identification result of driver turning intention is shown in Figure 10. The yaw rate, sideslip angle, lateral acceleration, and vehicle trajectory are shown in Figures 11-14. The comparison with the control experiment is presented in Table 3.

Figure 11 shows that the maximum yaw rate without control is $-35.517^{\circ} / \mathrm{s}$, whereas the reference yaw rate is $-18.66^{\circ} / \mathrm{s}$, indicative of poor vehicle stability. At $7.3 \mathrm{~s}$, the current initial stage of sharp turning intention is identified, whereupon the reference yaw rate is modified and the

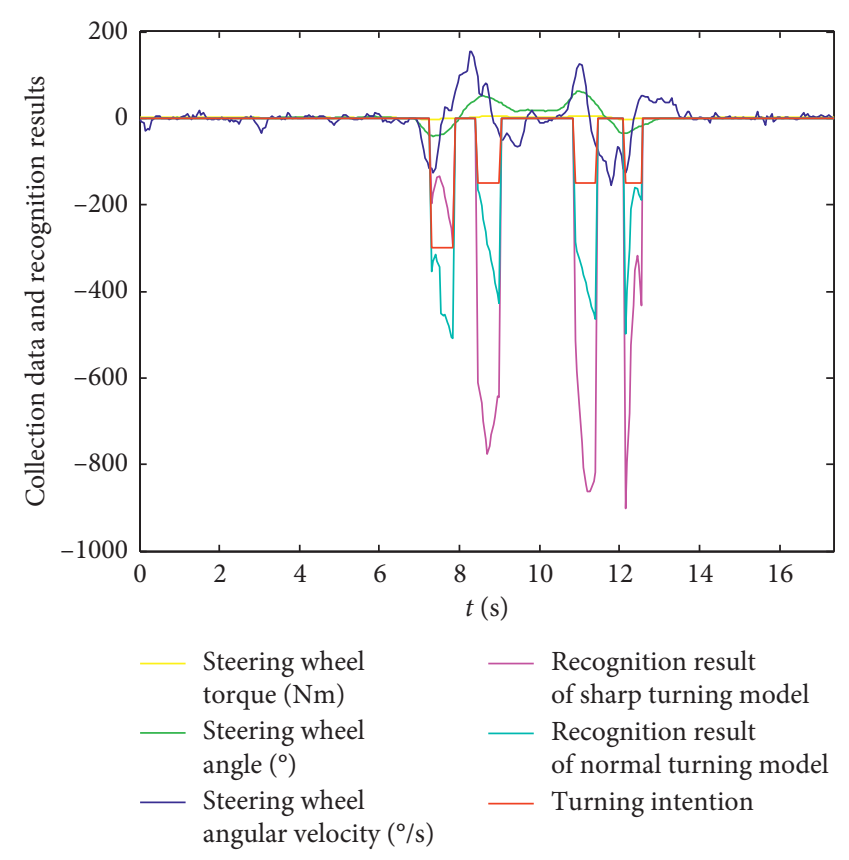

Figure 10: Driver turning intention recognition result.

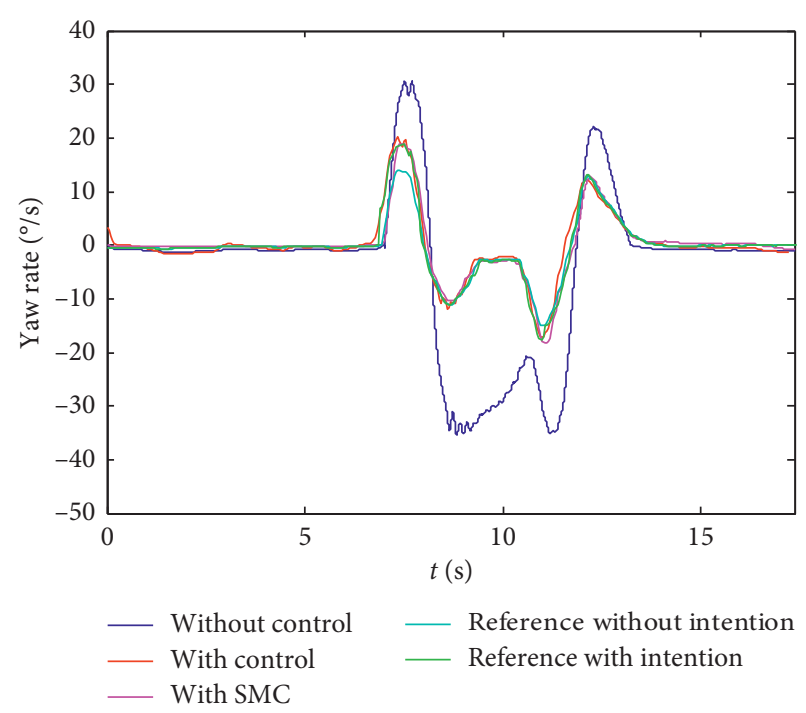

FIGURE 11: Yaw rate with control and without control.

stability control system activated. After $8.45 \mathrm{~s}$, the model identified that the current intention is normal turning, thus the reference yaw rate was no longer modified. As a result of the control provided by the vehicle stability system, the maximum yaw rate decreased by $47.22 \%$, to $18.744^{\circ} / \mathrm{s}$, the maximum sideslip angle decreased by $55.26 \%$, to $-4.391^{\circ}$, and the maximum lateral acceleration decreased by $19.1 \%$, to $4.638 \mathrm{~m} / \mathrm{s}^{2}$. Although dangerous conditions such as sideslip and spin without control did not arise, the actual sideslip angle and yaw rate are much higher than the reference. The stability system clearly reduces the sideslip angle and yaw rate to effectively improve the vehicle stability. Thus, the vehicle stability control system enables the vehicle to work 


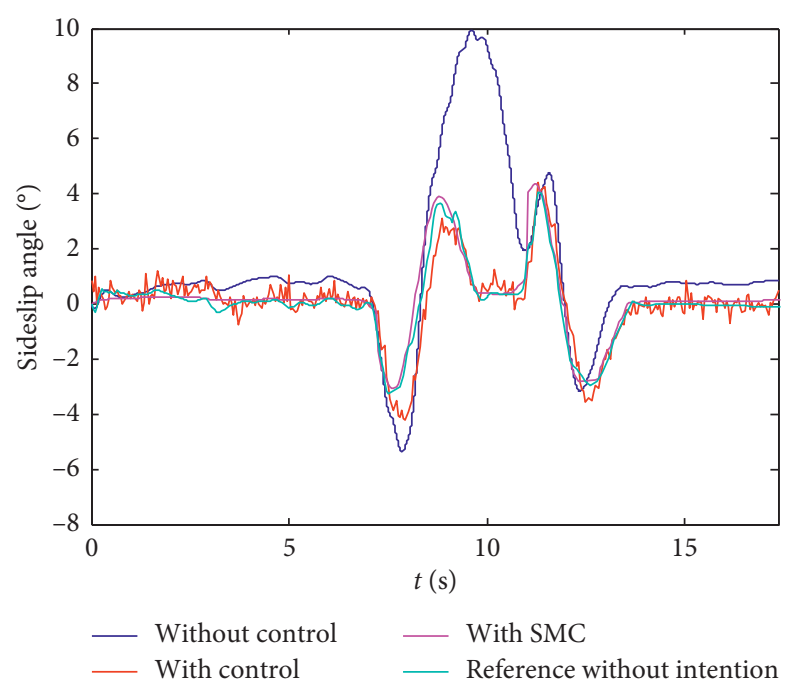

Figure 12: Sideslip angle with control and without control.

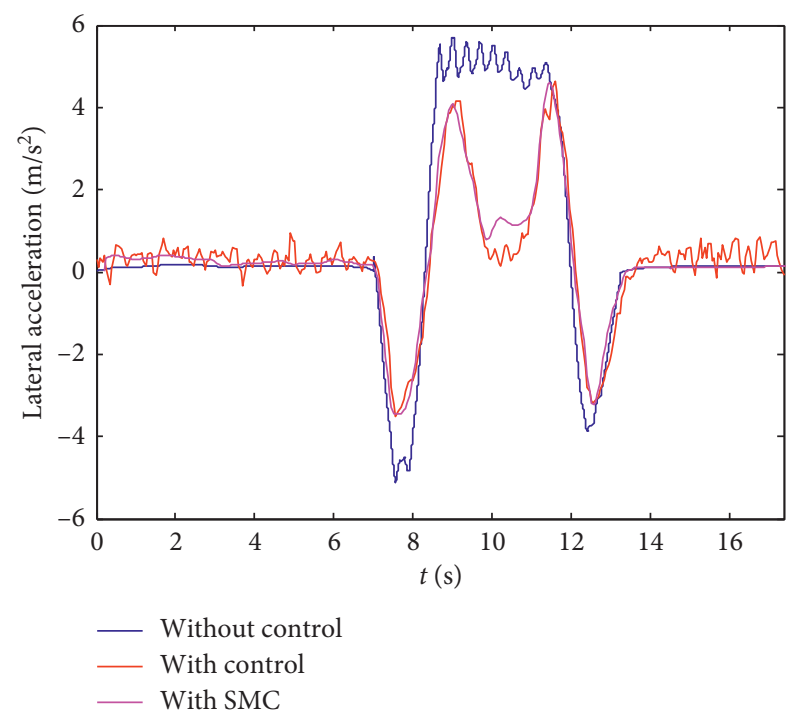

Figure 13: Lateral acceleration with control and without control.

much more smoothly. The actual driving trajectory is shown in Figure 14. Meanwhile, it can be seen from Table 3 that both the controller proposed in this paper and SMC with the vehicle reference model considering the driver's intention can track the control target well, and the control effect of some areas is better than that of the contrast controller.

5.2.2. Single-Lane Change Condition. The test was conducted by performing a single-lane change. The target driving path is shown in Figure 15. The adhesion coefficient of the test road was 0.4. First, the driver was required to keep the vehicle parallel with the road and keep the steering wheel facing forward. Subsequently, the driver controlled the

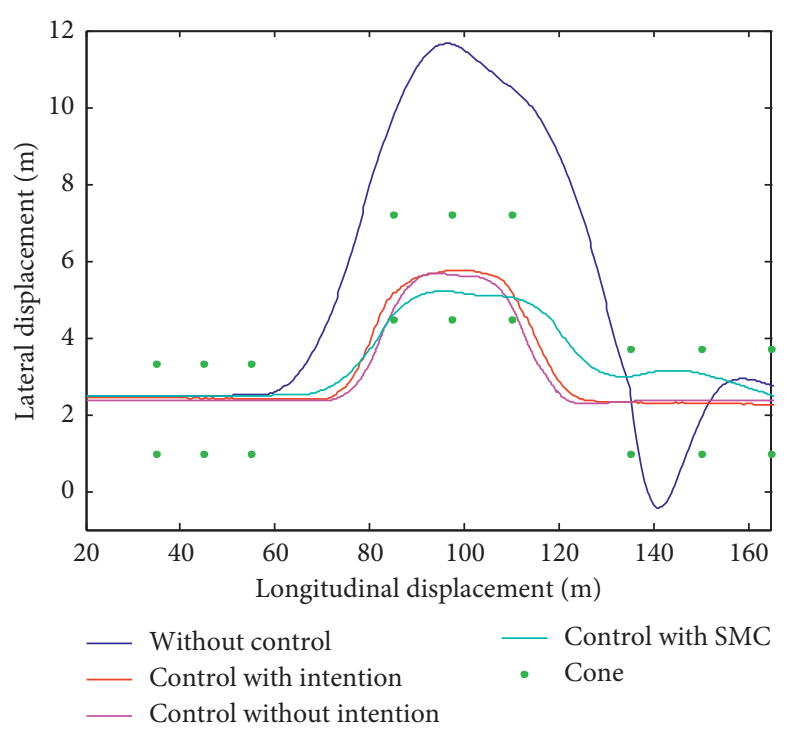

FIgURE 14: Vehicle track with control.

TABle 3: Control effect comparison.

\begin{tabular}{lccc}
\hline & Yaw rate & Sideslip angle & Lateral acceleration \\
\hline Without control & $-35.517^{\circ} / \mathrm{s}$ & $9.947^{\circ}$ & $5.733 \mathrm{~m} / \mathrm{s}^{2}$ \\
With control & $-18.744^{\circ} / \mathrm{s}$ & $4.391^{\circ}$ & $4.638 \mathrm{~m} / \mathrm{s}^{2}$ \\
With SMC & $-18.953^{\circ} / \mathrm{s}$ & $4.568^{\circ}$ & $4.895 \mathrm{~m} / \mathrm{s}^{2}$ \\
Reference & $-18.660^{\circ} / \mathrm{s}$ & $4.036^{\circ}$ & - \\
Decrease & $47.22 \%$ & $55.85 \%$ & $19.10 \%$ \\
\hline
\end{tabular}

vehicle to rapidly proceed ahead, such that the vehicle speed before entering the target path reached $40 \mathrm{~km} / \mathrm{h}$, during which time the steering wheel was not to be operated. The driver was then allowed to drive freely by following the cone track.

The identification result of driver turning intention is shown in Figure 16. The yaw rate, sideslip angle, lateral acceleration, and vehicle trajectory are shown in Figures 17-20. The comparison with the control effect is presented in Table 4.

Figures 17 and 18 show that the vehicle slips and spins after $7.5 \mathrm{~s}$ without stability control. The maximum yaw velocity reached $-62.779^{\circ} / \mathrm{s}$, and the maximum sideslip angle reached $70.740^{\circ}$, which are much more than the reference model. Thus, the vehicle stability control system was activated. Furthermore, the model identified that the current intention was normal turning; hence, the reference yaw rate was no longer modified. The vehicle stability system reduced the maximum yaw rate by $65.1 \%$, and the maximum sideslip angle by $92.7 \%$. The stability system therefore improved the vehicle stability effectively. The vehicle stability control system enables the vehicle to run much more smoothly in comparison, preventing dangerous conditions such as sideslip and spin. The actual driving 


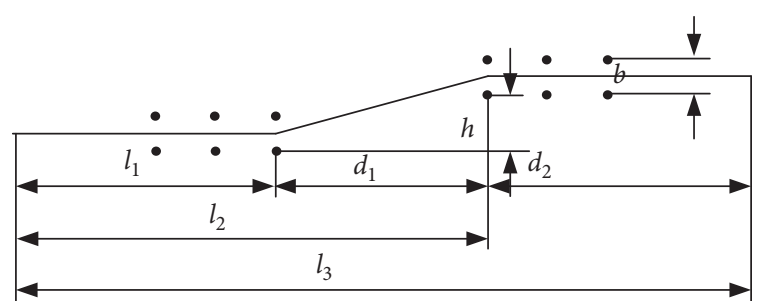

Figure 15: Schematic diagram for single-lane change test ( $h=3.5 \mathrm{~m} ; b=1.2 L+0.25 \mathrm{~m} ; L$ is the vehicle width; $d_{1}=50 \mathrm{~m}$; $\left.l_{2}=100 \mathrm{~m} ; l_{3}=15 u\right)$.

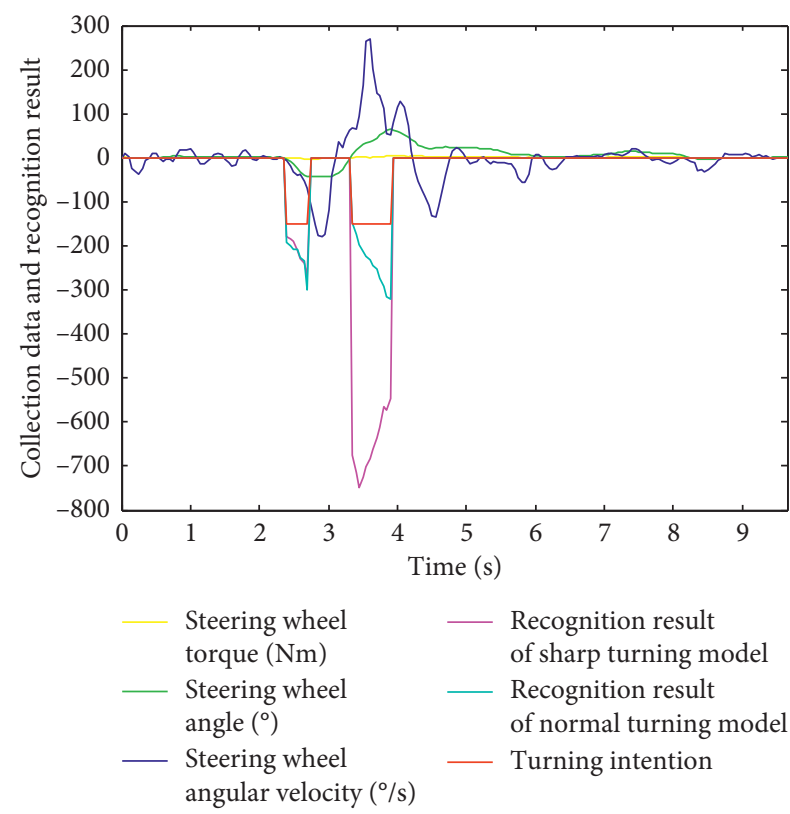

Figure 16: Driver turning intention recognition result.

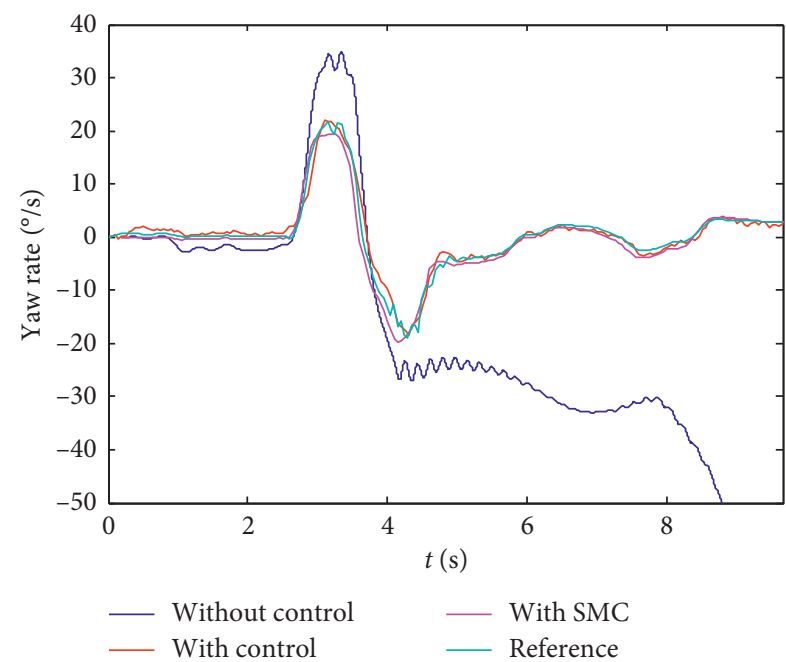

Figure 17: Yaw rate with control and without control.

trajectory is shown in Figure 20. Meanwhile, it can be seen from Table 4 that both the controller proposed in this paper and SMC with the vehicle reference model considering the

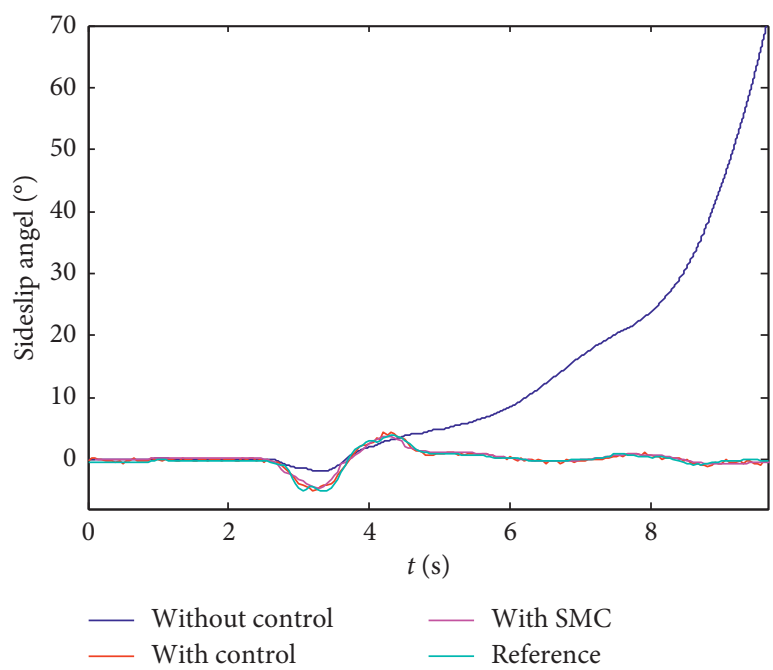

FIGURE 18: Sideslip angle with control and without control.

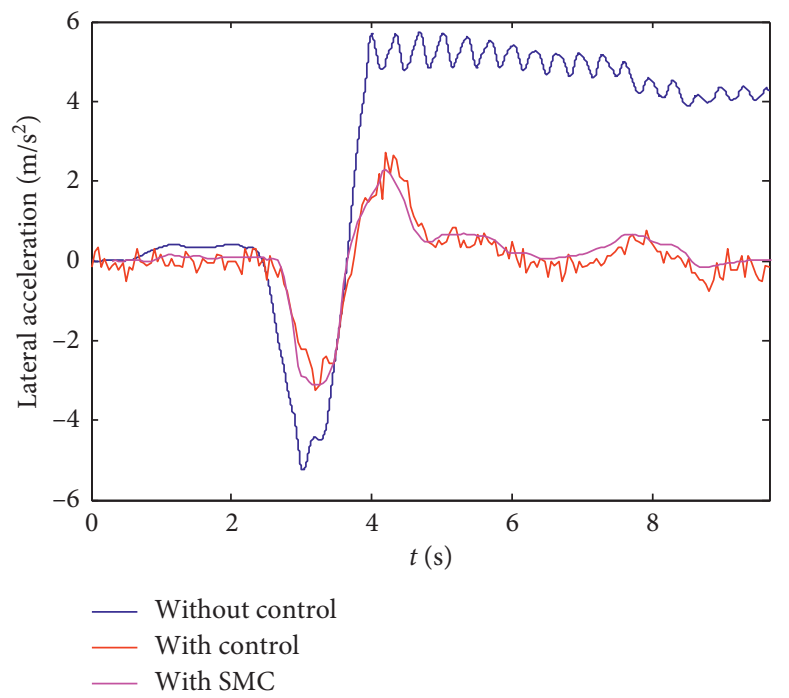

FIGURE 19: Lateral acceleration with control and without control.

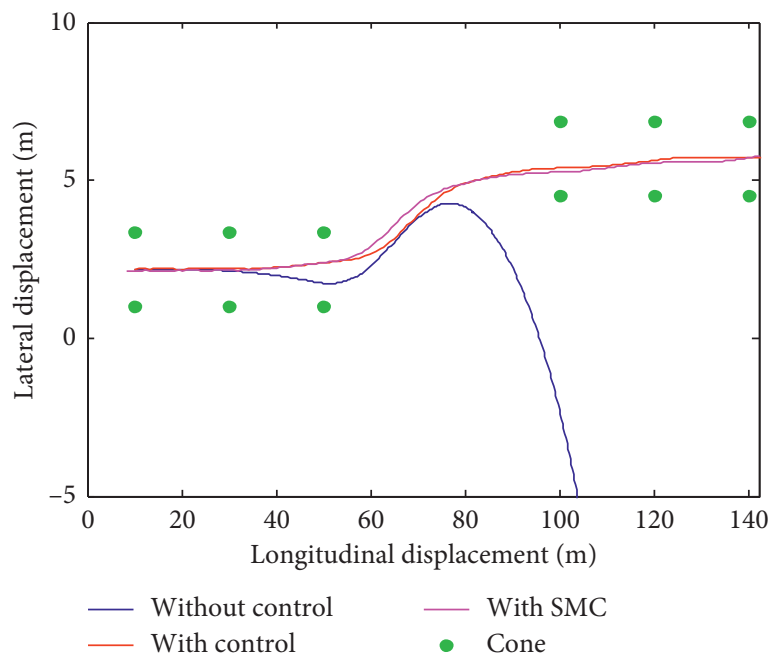

FIgURE 20: Vehicle track with control. 
TABLE 4: Control effect comparison.

\begin{tabular}{lccc}
\hline & Yaw rate & Sideslip angle & Lateral acceleration \\
\hline Without control & $-62.779^{\circ} / \mathrm{s}$ & $70.740^{\circ}$ & $5.761 \mathrm{~m} / \mathrm{s}^{2}$ \\
With control & $21.913^{\circ} / \mathrm{s}$ & $5.157^{\circ}$ & $2.705 \mathrm{~m} / \mathrm{s}^{2}$ \\
With SMC & $21.926^{\circ} / \mathrm{s}$ & $4.566^{\circ}$ & $3.124 \mathrm{~m} / \mathrm{s}^{2}$ \\
Reference & $21.76^{\circ} / \mathrm{s}$ & $5.208^{\circ}$ & - \\
Decrease & $65.1 \%$ & $92.7 \%$ & $53.04 \%$ \\
\hline
\end{tabular}

driver's intention can track the control target well, and the control effect of some areas is better than that of the contrast controller.

\section{Conclusion}

This paper proposes a vehicle stability control strategy considering the driver's turning intention for dual-motor drive electric vehicle.

(1) The upper controller of the hierarchical vehicle stability control system was constructed with the modified reference model as the control target. This model is modified by using turning urgency coefficient which is calculated on the basis of the recognition results of the GHMM/GGAP-RBF hybrid turning intention model. The lower layer controller takes the minimized sum of the square of operating working load rate of each tire as optimization objective and takes motor and road adhesion conditions as constraints to optimize the allocation additional yaw torque. The results show that the proposed vehicle stability control strategy can work satisfactorily and effectively improve the vehicle stability.

(2) Further studies could, apart from the turning intention, also consider the driver's acceleration intention, braking intention, and complex intention, to improve the stability control strategy, $X$-by-wire system, and advanced assisted driving system. This could be expected to improve the safety and comfort of vehicle operation.

\section{Nomenclature}

GHMM: Gaussian hidden Markov model

GGAP- Generalized growing and pruning radial basis

RBF: function

ABS: $\quad$ Antilock braking system

TCS: Traction control system

ECB: $\quad$ Electric control braking

EBD: $\quad$ Electric brake force distribution

AFS: $\quad$ Active front steering

$m: \quad$ Vehicle mass

$\omega_{\mathrm{r}}: \quad$ Yaw rate

$a, b$ : $\quad$ Distance from the center of mass to front and rear axle

$I_{s z z}: \quad \quad$ Moment of inertia of the vehicle around the $z$ axis

$\mu$ : $\quad$ Road adhesion coefficient

$a_{y}: \quad$ Lateral acceleration

\begin{tabular}{|c|c|}
\hline$a_{x q}:$ & Required longitudinal acceleration \\
\hline$\omega_{\text {rdes }}:$ & Ideal yaw rate \\
\hline$\omega_{\text {rref }}:$ & Reference yaw rate \\
\hline$\beta_{\mathrm{des}}:$ & Ideal sideslip angle \\
\hline$\beta_{\text {ref }}:$ & Reference sideslip angle \\
\hline$k_{1}, k_{2}:$ & Cornering stiffness of the front and rear axle \\
\hline$u, v:$ & Vehicle longitudinal and lateral speeds \\
\hline$\delta_{\mathrm{f}}:$ & Front wheel angle \\
\hline$i:$ & Transmission ratio of power train \\
\hline$\delta_{\text {wdes }}:$ & Ideal front wheel angle \\
\hline$\delta_{\text {swdes }}:$ & Ideal steering wheel angle \\
\hline$\rho:$ & Turning radius \\
\hline$i_{\mathrm{L}}:$ & Steering system angle ratio \\
\hline$V_{\mathrm{L}}:$ & Steering power-assisted factor \\
\hline$F_{z v}:$ & Static axle load \\
\hline$n_{v}:$ & Sum of pneumatic trail and king pin \\
\hline K: & Stability factor \\
\hline M: & Additional yaw moment \\
\hline$\Delta F_{x i j}:$ & Variation in longitudinal tire force \\
\hline$C_{i j}:$ & Cornering stiffness of tire \\
\hline$F_{x i j}:$ & Longitudinal tire force \\
\hline$F_{y i j}:$ & Lateral tire force \\
\hline$F_{z i j}:$ & Tire load \\
\hline$T_{d \max }:$ & Motor peak torque at different speeds \\
\hline$T_{\mathrm{e}}:$ & Motor peak torque \\
\hline$T_{b i j \max }:$ & Maximum braking force of each wheel \\
\hline$P_{\mathrm{e}}:$ & Maximum motor power \\
\hline$n_{N}:$ & Motor nom speed \\
\hline$n_{\max }:$ & Maximum motor speed \\
\hline$\eta:$ & Motor efficiency. \\
\hline
\end{tabular}

\section{Data Availability}

The data used to support the findings of this study are available from the corresponding author upon request.

\section{Conflicts of Interest}

The authors declare that they have no conflicts of interest.

\section{Acknowledgments}

This research was funded by National Key R\&D Program of China (no. 2018YFB1600700), China Postdoctoral Science Foundation (nos. 2018T111006 and 2017M613034), and Shaanxi Province Industrial Innovation Chain Project (no. 2018ZDCXL-GY-05-03-01).

\section{References}

[1] National Highway Traffic Safety Administration, Estimating Lives Saved by Electronic Stability Control, 2011-2015, National Highway Traffic Safety Administration, Washington, DC, USA, 2017.

[2] W. Jiang, Z. Yu, and L. Zhang, "A review on integrated chassis control," Automotive Engineering, vol. 29, no. 5, pp. 420-425, 2007.

[3] W. Chen, Q. Wang, and H. Xiao, Automobile System Dynamics and Integrated Control, Beijing Science Press, Beijing, China, 2014. 
[4] H. Zhang and J. Wang, "Vehicle lateral dynamics control through AFS/DYC and robust gain-scheduling approach," IEEE Transactions on Vehicular Technology, vol. 65, no. 1, pp. 489-494, 2016.

[5] C. Wang, C. Song, and J. Li, "Improvement of active yaw moment control based on electric-wheel vehicle ESC test platform," in Proceedings of the Fifth International Conference on Instrumentation and Measurement, Computer, Communication and Control, pp. 55-58, IEEE, New York, NY, USA, May 2016.

[6] J. Zhao, J. Huang, B. Zhu et al., "Nonlinear control of vehicle chassis planar stability based on T-S fuzzy model," in Proceedings of the SAE 2016 World Congress and Exhibition, Warrendale, PA, USA, April 2016.

[7] J. Xiao and T. Zhao, "Overview and prospect of T-S fuzzy control," Journal of Southwest Jiaotong University, vol. 51, no. 3, pp. 462-474, 2016.

[8] W. Zhao, X. Qin, and C. Wang, "Yaw and lateral stability control for four-wheel steer-by-wire system," IEEE/ASME Transactions on Mechatronics, vol. 23, no. 6, pp. 2628-2637, 2018.

[9] P. Hang, X. Chen, S. Fang, and F. Luo, "Robust control for four-wheel-independent-steering electric vehicle with steerby-wire system," International Journal of Automotive Technology, vol. 18, no. 5, pp. 785-797, 2017.

[10] W. Zhao and H. Zhang, "Coupling control strategy of force and displacement for electric differential power steering system of electric vehicle with motorized wheels," IEEE Transactions on Vehicular Technology, vol. 67, no. 9, pp. 8118-8128, 2018.

[11] W. Zhao, M. Fan, C. Wang, Z. Jin, and Y. Li, "H $\infty /$ extension stability control of automotive active front steering system," Mechanical Systems and Signal Processing, vol. 115, no. 115, pp. 621-636, 2019.

[12] Z. Wang, Y. Wang, L. Zhang et al., "Vehicle stability enhancement through hierarchical control for a four wheel independently actuated electric vehicle," Energies, vol. 10, no. 7, 2017.

[13] H. Chen, Model Predictive Control, Beijing Science Press, Beijing, China, 2013.

[14] M. Jalali, A. Khajepour, S.-k. Chen, and B. Litkouhi, "Integrated stability and traction control for electric vehicles using model predictive control," Control Engineering Practice, vol. 54, pp. 256-266, 2016.

[15] O. Barbarisi, G. Palmieri, S. Scala et al., "LTV-MPC for yaw rate control and side slip control with dynamically constrained differential braking," European Journal of Control, vol. 15, no. 3-4, pp. 468-479, 2009.

[16] F. Paolo, H. E. Tseng, B. Francesco et al., "MPC-based yaw and lateral stabilisation via active front steering and braking," Vehicle System Dynamics, vol. 46, no. sup1, pp. 611-628, 2008.

[17] S. Wang, X. Zhao, Q. Yu et al., "Research on strategy of the stability control system of dual-motor drive electric vehicle," in Proceedings of the IEEE International Symposium on Circuits and Systems (ISCAS), Sapporo, Japan, May 2019.

[18] Z. Yu, P. Yang, and X. Lu, "Application of control allocation in distributed drive electric vehicle," Journal of Mechanical Engineering, vol. 50, no. 18, pp. 99-107, 2014.

[19] D. Yin, D. Shan, and B. C. Chen, "A torque distribution approach to electronic stability control for in-wheel motor electric vehicles," in Proceedings of the International Conference on Applied System Innovation, pp. 1-4, IEEE, Osaka, Japan, May 2016.
[20] L. Zhai, T. Sun, and J. Wang, "Electronic stability control based on motor driving and braking torque distribution for a four in-wheel motor drive electric vehicle," IEEE Transactions on Vehicular Technology, vol. 65, no. 6, pp. 4726-4739, 2016.

[21] J. Park, H. Jeong, I. Jang, and S.-H. Hwang, "Torque distribution algorithm for an independently driven electric vehicle using a fuzzy control method," Energies, vol. 8, no. 8, pp. 8537-8561, 2015.

[22] D. Kim and H. Kim, "Vehicle stability control with regenerative braking and electronic brake force distribution for a four-wheel drive hybrid electric vehicle," Proceedings of the Institution of Mechanical Engineers, Part D: Journal of Automobile Engineering, vol. 220, no. 6, pp. 683-693, 2006.

[23] Q. Wang, L. Sun, X. Tang et al., "A study on braking intention identification for HEV," Automotive Engineering, vol. 9, pp. 769-831, 2013.

[24] G. Ma, Z. Liu, X. Pei et al., "Identification of cut-in maneuver of side lane vehicles based on fuzzy support vector machines," Automotive Engineering, vol. 36, no. 3, pp. 316-320, 2014.

[25] W. Zhao, Development and Test of Intention Recognition and Braking Force Distribution Control Strategies for TractorSemitrailer, JiLin University, Changchun, China, 2013.

[26] L. Xiong, G. W. Teng, Z. P. Yu, W. X. Zhang, and Y. Feng, "Novel stability control strategy for distributed drive electric vehicle based on driver operation intention," International Journal of Automotive Technology, vol. 17, no. 4, pp. 651-663, 2016.

[27] S. Wang, Q. Yu, and X. Zhao, "Study on driver's turning intention recognition hybrid model of GHMM and GGAPRBF neural network," Advances in Mechanical Engineering, vol. 10, no. 3, pp. 1-16, 2018.

[28] H. Berndt, J. Emmert, K. Ditemater et al., "Continuous driver intention recognition with hidden markov models," in Proceedings of the 11th International IEEE Conference on Intelligent Transportation Systems, Beijing, China, October 2008.

[29] X. Zheng, The Study of Cough Signal Recognition Based on HMM-ANN Hybrid Model, Chongqing University, Chongqing, China, 2011.

[30] L. He, B. Ma, and C. Zong, "Emergency steering control based on driver steering intention recognition for steer-by-wire vehicle," Journal of Hunan University (Natural Sciences), vol. 1, pp. 81-86, 2014.

[31] R. Pongsathorn, M. Takuya, and N. Masao, "Direct yaw moment control system based on driver behavior recognition," Vehicle System Dynamics, vol. 46, pp. 911-921, 2008.

[32] G.-B. Huang, P. Saratchandran, and N. Sundararajan, "An efficient sequential learning algorithm for growing and pruning RBF (GAP-RBF) networks," IEEE Transactions on Systems, Man and Cybernetics, Part B (Cybernetics), vol. 34, no. 6, pp. 2284-2292, 2004.

[33] Y. Lu Yingwei, N. Sundararajan, and P. Saratchandran, "Performance evaluation of a sequential minimal radial basis function (RBF) neural network learning algorithm," IEEE Transactions on Neural Networks, vol. 9, no. 2, pp. 308-318, 1998.

[34] G. B. Huang, P. Saratchandran, and N. Sundararajan, "A generalized growing and pruning RBF (GGAP-RBF) neural network for function approximation," IEEE Transactions on Neural Networks, vol. 16, no. 16, pp. 57-67, 2005.

[35] V. Kadirkamanathan and M. Niranjan, "A function estimation approach to sequential learning with neural networks," Neural Computation, vol. 5, no. 6, pp. 954-975, 1993.

[36] Z. Yu, Automobile Theory, China Machine Press, Beijing, China, 5th edition, 2010. 
[37] Y. Chen and Q. Yu, Automotive Dynamics, Tsinghua University Press, Beijing, China, 4th edition, 2009.

[38] F. Huang, The Acceleration and Deceleration Algorithm and Simulation of Position and Pressure Control Conversion Process of Electro-Hydraulic Servo System, Wuhan University of Science and Technology, Wuhan, China, 2011.

[39] D. Q. Mayne, J. B. Rawlings, C. V. Rao, and P. O. M. Scokaert, "Constrained model predictive control: stability and optimality," Automatica, vol. 36, no. 6, pp. 789-814, 2000.

[40] L. Liu, Nonlinear Analysis and Control Strategy Evaluation on the Stability of Vehicle 3-DOF Planar Motion, JiLin University, Changchun, China, 2010.

[41] S. Wang, Research on Vehicle Stability Control System for Dual-Motor Drive Electric Vehicle, Chang'an University, Xi'an, China, 2018.

[42] Q. Jin and Z. Zhong, "Analysis of electric braking characteristics and control strategy of electric vehicle," Shang Hai Automobile, no. 2, pp. 32-34, 2003.

[43] ISO 3888-2:2011, Passenger Cars-Test Track for a Severe LaneChange Manoeuvre-Part 2: Obstacle Avoidance, International Organization for Standardization, Geneva, Switzerland, 2011. 


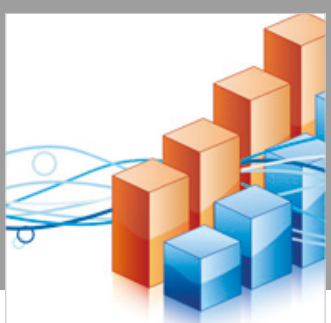

Advances in

Operations Research

\section{-n-m}
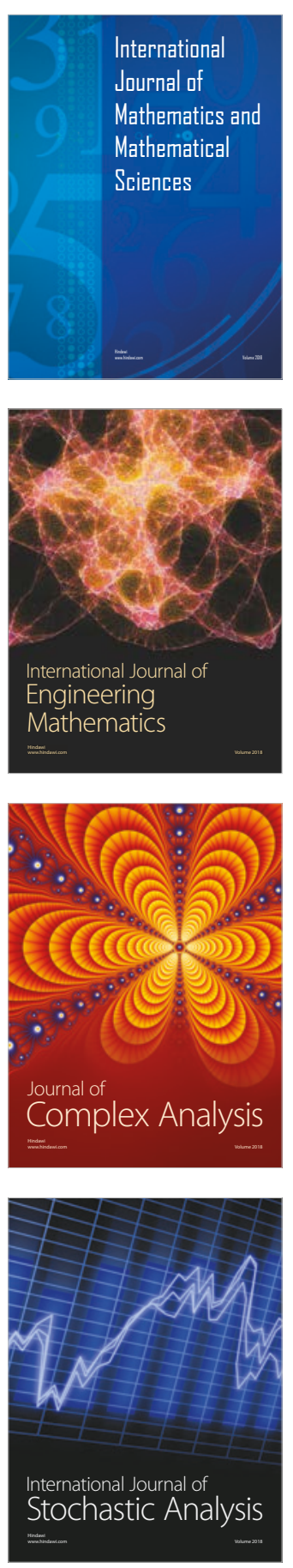
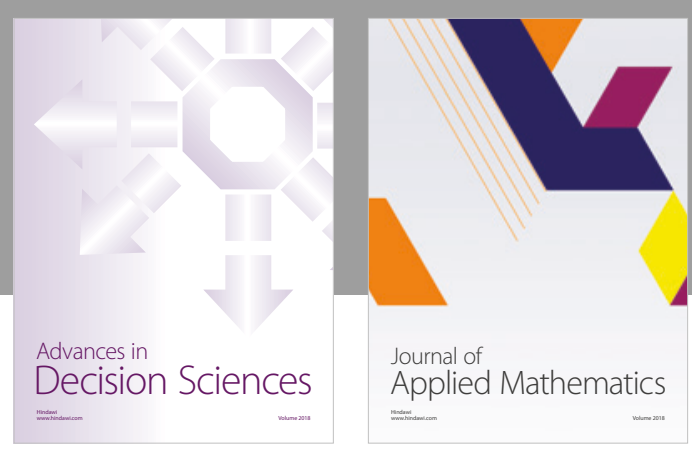

Journal of

Applied Mathematics
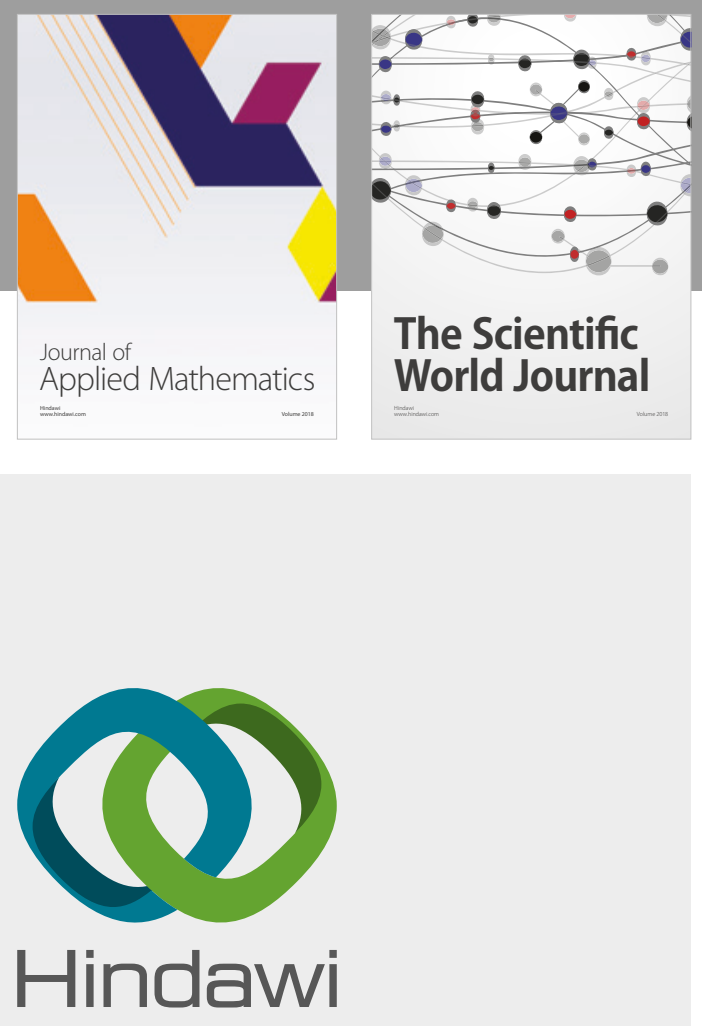

Submit your manuscripts at

www.hindawi.com

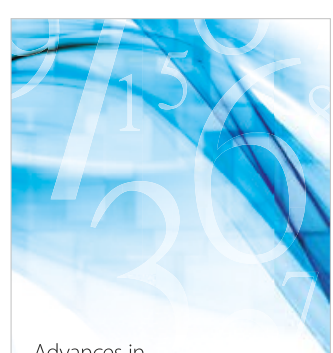

Advances in
Numerical Analysis
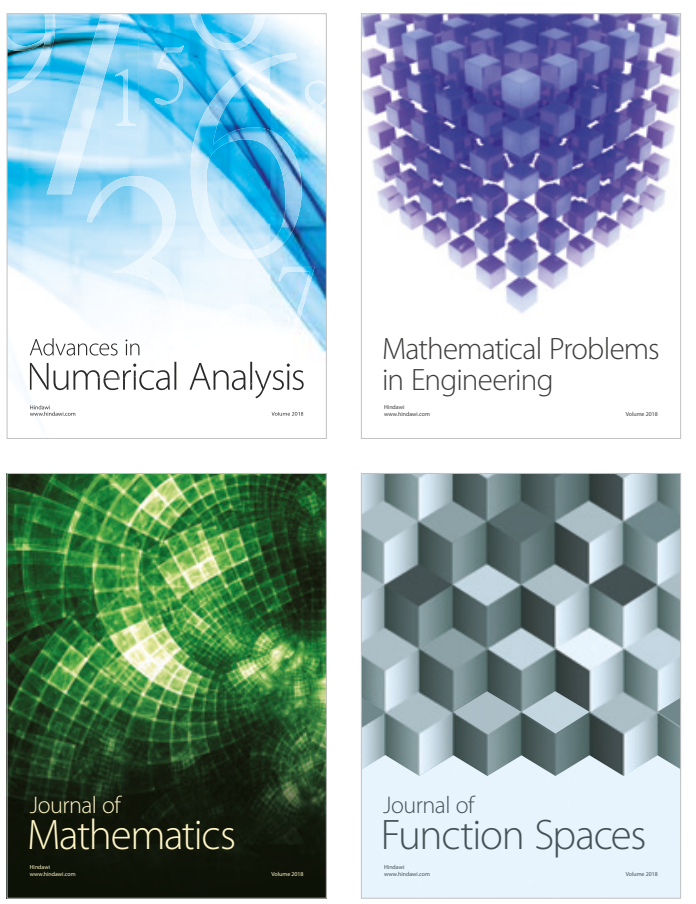

Mathematical Problems in Engineering

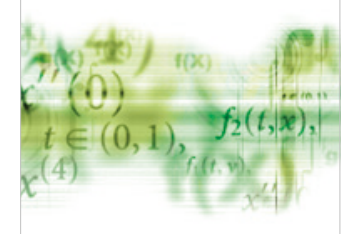

International Journal of

Differential Equations

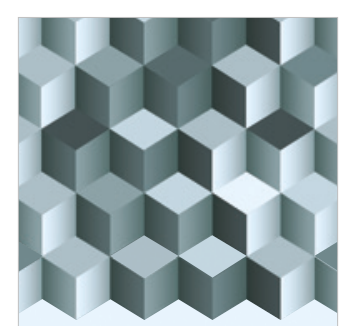

Journal of

Function Spaces

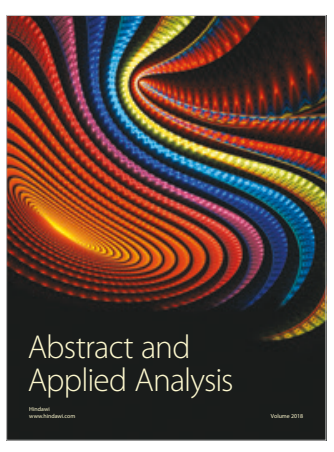

The Scientific

World Journal

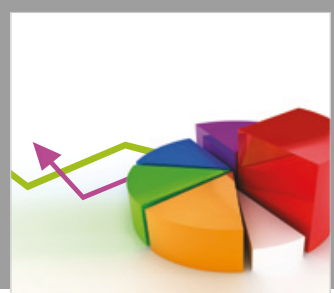

Journal of

Probability and Statistics
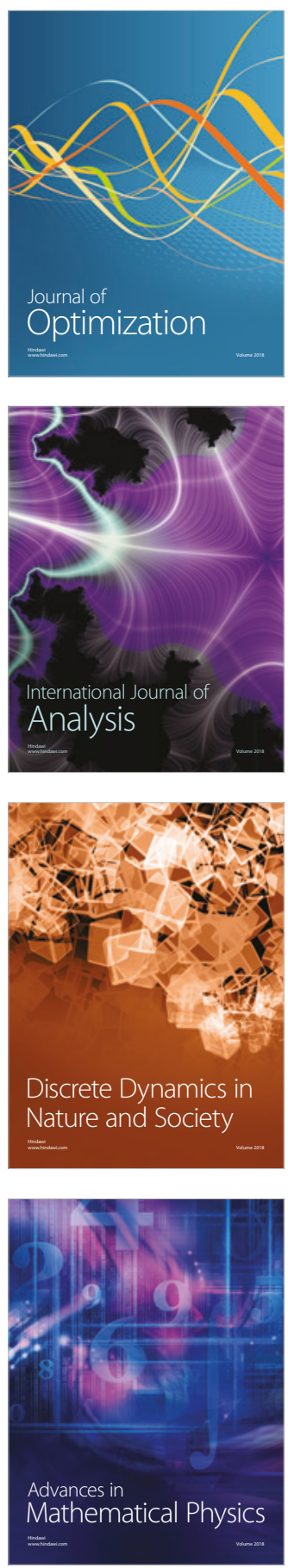